\title{
Inhibition of oxidative stress by coenzyme Q10 increases mitochondrial mass and improves bioenergetic function in optic nerve head astrocytes
}

\author{
YH Noh ${ }^{1,4}$, K-Y Kim ${ }^{2,4}$, MS Shim ${ }^{1}$, S-H Choi ${ }^{3}$, S Choi ${ }^{2}$, MH Ellisman ${ }^{2}$, RN Weinreb ${ }^{1}$, GA Perkins ${ }^{2}$ and W-K Ju*,1
}

Oxidative stress contributes to dysfunction of glial cells in the optic nerve head (ONH). However, the biological basis of the precise functional role of mitochondria in this dysfunction is not fully understood. Coenzyme $Q 10\left(C_{0} Q_{10}\right)$, an essential cofactor of the electron transport chain and a potent antioxidant, acts by scavenging reactive oxygen species (ROS) for protecting neuronal cells against oxidative stress in many neurodegenerative diseases. Here, we tested whether hydrogen peroxide $\left(100 \mu \mathrm{M} \mathrm{H}_{2} \mathrm{O}_{2}\right.$ )-induced oxidative stress alters the mitochondrial network, oxidative phosphorylation (OXPHOS) complex (Cx) expression and bioenergetics, as well as whether $\mathrm{CoQ}_{10}$ can ameliorate oxidative stress-mediated alterations in mitochondria of the $\mathrm{ONH}$ astrocytes in vitro. Oxidative stress triggered the activation of $\mathrm{ONH}$ astrocytes and the upregulation of superoxide dismutase 2 (SOD2) and heme oxygenase-1 (HO-1) protein expression in the ONH astrocytes. In contrast, $\mathrm{CoQ}_{10}$ not only prevented activation of $\mathrm{ONH}$ astrocytes but also significantly decreased SOD2 and HO-1 protein expression in the ONH astrocytes against oxidative stress. Further, $\mathrm{CoQ}_{10}$ prevented a significant loss of mitochondrial mass by increasing mitochondrial number and volume density and by preserving mitochondrial cristae structure, as well as promoted mitofilin and peroxisome-proliferator-activated receptor- $\gamma$ coactivator-1 protein expression in the $\mathrm{ONH}$ astrocyte, suggesting an induction of mitochondrial biogenesis. Finally, oxidative stress triggered the upregulation of OXPHOS Cx protein expression, as well as reduction of cellular adeonsine triphosphate (ATP) production and increase of ROS generation in the ONH astocytes. However, $\mathrm{CoQ}_{10}$ preserved OXPHOS protein expression and cellular ATP production, as well as decreased ROS generation in the ONH astrocytes. On the basis of these observations, we suggest that oxidative stress-mediated mitochondrial dysfunction or alteration may be an important pathophysiological mechanism in the dysfunction of $\mathrm{ONH}$ astrocytes. $\mathrm{CoQ}_{10}$ may provide new therapeutic potentials and strategies for protecting $\mathrm{ONH}$ astrocytes against oxidative stress-mediated mitochondrial dysfunction or alteration in glaucoma and other optic neuropathies.

Cell Death and Disease (2013) 4, e820; doi:10.1038/cddis.2013.341; published online 3 October 2013

Subject Category: Neuroscience

Impaired mitochondrial oxidative phosphorylation (OXPHOS) generates excessive reactive oxygen species (ROS), leading to mitochondrial damage, oxidative stress and apoptotic cell death. ${ }^{1,2}$ Increasing evidence demonstrates that oxidative stress links to mitochondrial dysfunction in glaucomatous neurodegeneration. ${ }^{2-5} \mathrm{~A}$ recent study reported that lamina cribrosa cells in the optic nerve head $(\mathrm{ONH})$ from glaucomatous human patients showed increased ROS production, impaired mitochondrial function and elevated cytosolic $\mathrm{Ca}^{2+},{ }^{3}$ suggesting that a cycle of oxidative stress, mitochondrial dysfunction and dysregulation of calcium homeostasis may contribute to the pathogenesis of $\mathrm{ONH}$ degeneration in glaucoma. ${ }^{3}$ Although alteration of antioxidant enzymes has been implicated in glaucomatous $\mathrm{ONH}$ astrocytes, ${ }^{6}$ it remains unknown whether oxidative stress alters the mitochondrial network and triggers bioenergetic dysfunction in the $\mathrm{ONH}$ astrocytes.

Glaucoma is the leading cause of irreversible blindness and affects 70 million people worldwide. ${ }^{7}$ Although elevated intraocular pressure (IOP) is an important risk factor for $\mathrm{ONH}$ degeneration and retinal ganglion cell (RGC) death in glaucoma, lowering IOP is not always effective for preserving

\footnotetext{
${ }^{1}$ Laboratory for Optic Nerve Biology, Hamilton Glaucoma Center and Department of Ophthalmology, University of California, San Diego, La Jolla, CA, USA; ${ }^{2}$ National Center for Microscopy and Imaging Research and Department of Neurosciences, University of California, San Diego, La Jolla, CA, USA and ${ }^{3}$ Department of Medicine, University of California, San Diego, La Jolla, CA, USA

*Corresponding author: W-K Ju, Laboratory for Optic Nerve Biology, Hamilton Glaucoma Center, Department of Ophthalmology, University of California, San Diego, 9415 Campus Point Drive, La Jolla, CA 92037, USA. Tel: 858246 0452; Fax: 858822 4717; E-mail: danielju@ glaucoma.ucsd.edu

${ }^{4}$ These authors contributed equally to this work.

Keywords: oxidative stress; optic nerve head astrocytes; mitochondrial dysfunction; coenzyme Q10; glaucoma; OXPHOS complex

Abbreviations: ATP, adeonsine triphosphate; BSA, bovine serum albumin; $\mathrm{COQ}_{10}$, coenzyme Q10; CM- $\mathrm{H}_{2} \mathrm{DCFDA}, 5$-(and-6)-chloromethyl-2',7'-dichlorodihydrofluorescein diacetate, acetyl ester; Cx, complex; DMEM, Dulbecco's modified Eagle's medium; DPBS, Dulbecco's phosphate-buffered saline; FBS, fetal bovine serum; GFAP, glial fibrillary acidic protein; $\mathrm{H}_{2} \mathrm{O}_{2}$, hydrogen peroxide; HO-1, heme oxygenase-1; IOP, intraocular pressure; MBP, myelin basic protein; MTT, 3-[4,5-dimethylthiazol-2yl]-2,5-diphenyl tetrazolium bromide; ONH, optic nerve head; OXPHOS, oxidative phoshorylation; PBST, 0.1\% Tween-20 in PBS; PGC-1 $\alpha$, peroxisome-proliferator-activated receptor- $\gamma$ coactivator-1; RGC, retinal ganglion cell; ROS, reactive oxygen species; SA, surface area; SOD2, superoxide dismutase 2 Received 07.3.13; revised 02.7.13; accepted 07.8.13; Edited by A Finazzi-Agró
} 
visual function in patients. ${ }^{7}$ Alterations in astrocytes have been implicated as important pathophysiological mechanisms in glaucomatous ONH degeneration. ${ }^{8-10}$ However, the biological basis of the precise functional role of $\mathrm{ONH}$ astrocytes in glaucomatous neurodegeneration is not fully understood.

Since the first evidence of impaired mitochondrial respiration-mediated mitochondrial dysfunction in patients with primary open-angle glaucoma, ${ }^{4}$ we have found that mitochondrial dysfunction is associated with $\mathrm{ONH}$ degeneration and RGC death in a mouse model of glaucoma, ${ }^{11-13}$ suggesting a distinct mitochondrial dysfunction-mediated degenerative pathway in glaucomatous neurodegeneration. Growing evidence indicates that glaucomatous damage, including elevated IOP, contributes to dysfunction of glial cells or loss of astrocytic processes in the $\mathrm{ONH}$ of rodent models of glaucoma. ${ }^{10,14}$ Regardless, the relationship between mitochondrial dysfunction and $\mathrm{ONH}$ astrocytes in glaucomatous neurodegeneration remain unknown.

Coenzyme $\mathrm{Q} 10\left(\mathrm{CoQ}_{10}\right)$, an essential cofactor of the electron transport chain, acts by maintaining the mitochondrial membrane potential, supporting adenosine triphosphate (ATP) synthesis and inhibiting ROS generation, thus protecting neuronal cells against oxidative stress in neurodegenerative diseases. ${ }^{15-17}$ Of note, the levels of $\mathrm{CoQ}_{10}$ in the human retina can decline by $\sim 40 \%$ with age, ${ }^{18}$ raising the possibility that this decline of $\mathrm{CoQ}_{10}$ may contribute to age-related ROS generation in glaucomatous $\mathrm{ONH}$ neurodegeneration. Previous studies demonstrated that $\mathrm{CoQ}_{10}$ protects retinal cells against oxidative stress in vitro and in vivo, as well as prevents retinal damage induced by acute IOP elevation or excitotoxicity in vivo. ${ }^{19-22}$

Here, we tested whether hydrogen peroxide $\left(\mathrm{H}_{2} \mathrm{O}_{2}\right)$ induced oxidative stress alters the mitochondrial network, bioenergetics and OXPHOS complex (Cx) expression, and whether $\mathrm{CoQ}_{10}$ can ameliorate oxidative stress-mediated alterations in mitochondria of $\mathrm{ONH}$ astrocytes in vitro.

\section{Results}

$\mathrm{CoQ}_{10}$ ameliorates oxidative stress in the $\mathrm{ONH}$ astrocytes. Pure $\mathrm{ONH}$ astrocytes were generated from postnatal day 5 Sprague-Dawley rats using a modification of a previous protocol by Hernandez et al. ${ }^{23}$ As shown in Figure 1, the cells migrated from cultured $\mathrm{ONH}$ tissues were purified by vigorous shaking and serum deprivation (Figure 1a). To confirm whether purified cells were astrocytes, we performed Western blot and immunocytochemical analyses using markers for astrocytes, microglial cells and oligodendrocytes. We found that the purified cells were positive for glial fibrillary acidic protein (GFAP), a marker for astrocytes, but were negative for Iba1, a marker for microglial cells, and myelin basic protein (MBP), a marker for oligodendrocytes (Figure 1b). In contrast, the protein extract from rat $\mathrm{ON}$ tissues contained all four of markers. Immunocytochemistry showed a strong immunoreacitivity for GFAP in cultured $\mathrm{ONH}$ astrocytes (Figure 1b), suggesting that the purified cells from $\mathrm{ONH}$ tissues were astrocytes.

As reduction of oxidative stress has been proposed to be protective against glaucomatous damage, ${ }^{24,25}$ we determined whether $\mathrm{CoQ}_{10}$ treatment prevents oxidative stress-mediated activation of $\mathrm{ONH}$ astrocytes in vitro using antibodies for GFAP, superoxide dismutase 2 (SOD2) and heme oxygenase-1 (HO-1). We observed that GFAP protein expression was significantly increased by $1.23 \pm 0.10$-fold in the $\mathrm{ONH}$ astrocytes exposed to $\mathrm{H}_{2} \mathrm{O}_{2}(100 \mu \mathrm{M})$ compared with control cells exposed to vehicle. In contrast, $\mathrm{CoQ}_{10}$ significantly reduced GFAP protein expression by $0.97 \pm 0.08$-fold in the $\mathrm{ONH}$ astrocytes exposed to $\mathrm{H}_{2} \mathrm{O}_{2}$ (Figure 2a). Intriguingly, we also found that SOD2 and $\mathrm{HO}-1$ protein expression was significantly increased by $1.40 \pm 0.13$ - and $1.2 \pm 0.14$-fold in the $\mathrm{ONH}$ astrocytes exposed to $\mathrm{H}_{2} \mathrm{O}_{2}$ compared with control cells, respectively. In contrast, $\mathrm{CoQ}_{10}$ significantly reduced SOD2 and $\mathrm{HO}-1$ protein expression by $0.63 \pm 0.11$ - and $0.63 \pm 0.07$-fold in the $\mathrm{ONH}$ astrocytes exposed to $\mathrm{H}_{2} \mathrm{O}_{2}$, respectively (Figure 2a). To determine whether oxidative stress alters SOD2 distribution and expression in the $\mathrm{ONH}$ astocytes, we performed immunocytochemistry in cultured $\mathrm{ONH}$ astrocytes using the antibody for SOD2. Consistently, we found that SOD2 immunoreactivity was present in the mitochondria of the $\mathrm{ONH}$ astrocytes. Moreover, we observed that $\mathrm{ONH}$ astrocytes exposed to $\mathrm{H}_{2} \mathrm{O}_{2}$ showed increase of SOD2 immunoreactivity in the mitochondria and cytoplasm compared with control cells. In contrast, $\mathrm{CoQ}_{10}$ decreased SOD2 immunoreactivity in the $\mathrm{ONH}$ astrocytes exposed to $\mathrm{H}_{2} \mathrm{O}_{2}$ (Figure $2 \mathrm{~b}$ ). These results indicate that $\mathrm{CoQ}_{10}$ prevents activation of $\mathrm{ONH}$ astrocytes by blocking oxidative stress.

$\mathrm{CoQ}_{10}$ triggers mitochondrial biogenesis against oxidative stress. To determine whether oxidative stress triggers alteration of the intracellular mitochondrial network in $\mathrm{ONH}$ astrocytes and whether $\mathrm{CoQ}_{10}$ treatment inhibits this alteration in mitochondria against oxidative stress, the mitochondrial morphology of $\mathrm{ONH}$ astrocytes were assessed by MitoTracker Red (Invitrogen-Molecular Probes, Eugene, OR, USA) staining, a marker for mitochondria. Further, we quantified the aterations of mitochondrial number, length and volume density following exposure of $\mathrm{H}_{2} \mathrm{O}_{2}$ using transmission electron microscopy (TEM) analysis. Our results showed that control $\mathrm{ONH}$ astrocytes contained classic elongated tubular mitochondria. However, $\mathrm{ONH}$ astrocytes exposed to $\mathrm{H}_{2} \mathrm{O}_{2}$ contained small rounded mitochondria. Interestingly, $\mathrm{ONH}$ astrocytes pretreated with $\mathrm{CoQ}_{10}$ and exposed to $\mathrm{H}_{2} \mathrm{O}_{2}$ showed a partial preservation of mitochondrial morphology compared with the $\mathrm{ONH}$ astrocytes exposed to $\mathrm{H}_{2} \mathrm{O}_{2}$ (Figure 3). Of note, representative 2D images from TEM showed that $\mathrm{ONH}$ astrocytes exposed to oxidative stress produced fewer mitochondria. In good agreement with this result, quantitative analyses importantly showed that the number of mitochondria, normalized to the total area occupied by somas in each image, was significantly decreased in the $\mathrm{ONH}$ astrocytes exposed to $\mathrm{H}_{2} \mathrm{O}_{2}$ $\left(0.15 \pm 0.01 \mu \mathrm{m}^{2}\right)$ compared with control $\mathrm{ONH}$ astrocytes $\left(0.52 \pm 0.07 \mu \mathrm{m}^{2} ; P<0.001\right.$; Figures $4 \mathrm{a}$ and $\left.\mathrm{b}\right)$. In contrast, pretreatment of $\mathrm{CoQ}_{10}$ increased mitochondrial numbers to a lesser extent $(0.26 \pm 0.03)$ compared with $\mathrm{ONH}$ astrocytes exposed to $\mathrm{H}_{2} \mathrm{O}_{2}(P<0.05$; Figure $4 \mathrm{~b})$. In addition, mitochondrial volume density, defined as the volume occupied by mitochondria divided by the volume occupied by the cytoplasm in terms of a percentage, was decreased in the $\mathrm{ONH}$ astrocytes exposed to $\mathrm{H}_{2} \mathrm{O}_{2}(3.58 \pm 0.38 \%)$ compared 
b

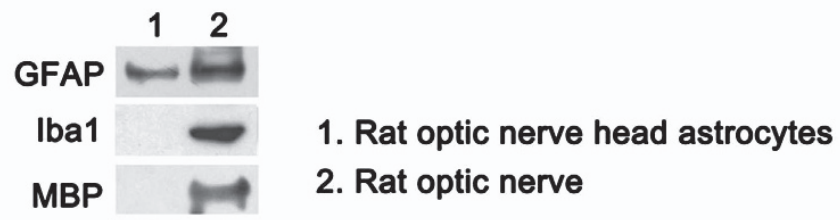

Actin GFAP Nucleus

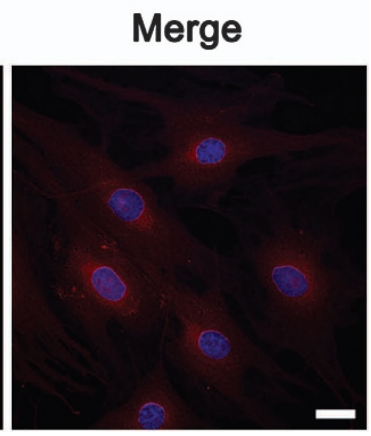

Figure 1 Characterization of cultured rat optic nerve head (ONH) astrocytes. (a) The diagram represents purification of ONH astrocytes from rats. (b) The protein extracts from ONH astrocytes were positive for GFAP, a marker for astrocytes but were negative for lba1, a marker for microglial cells, and MBP, a maker for oligodendrocytes. The protein extract from rat optic nerve tissue was used for positive control. A representative image shows that GFAP immunoreactivity is present in the ONH astrocytes. GFAP, glial fibrillary acidic protein; MBP, myelin basic protein. Scale bar, $20 \mu \mathrm{m}$

with control $\mathrm{ONH}$ astrocytes $(6.36 \pm 0.87 \% ; P<0.01$; Figures $4 a$ and $b)$. In contrast, pretreatment of $\mathrm{CoQ}_{10}$ significantly increased the mitochondrial volume density $(5.84 \pm 0.75 \%)$ compared with $\mathrm{ONH}$ astrocytes exposed to $\mathrm{H}_{2} \mathrm{O}_{2}(P<0.05$; Figure 4b). Interestingly, however, there was no difference in mitochondrial length among control, $\mathrm{H}_{2} \mathrm{O}_{2}-$ and $\mathrm{CoQ}_{10} / \mathrm{H}_{2} \mathrm{O}_{2}-$ treated $\mathrm{ONH}$ astrocytes (Figures $4 \mathrm{a}$ and $\mathrm{b}$ ), indicating that mitochondrial biogenesis by $\mathrm{CoQ}_{10}$ did not come from increased mitochondrial fusion but rather by nascent mitochondria.

$\mathrm{CoQ}_{10}$ promotes mitofilin and PGC-1 $\alpha$ protein expression against oxidative stress. The mitochondrial inner membrane protein mitofilin is critical for maintenance of mitochondrial cristae morphology and is reduced following oxidative stress. ${ }^{26,27}$ To determine whether $\mathrm{H}_{2} \mathrm{O}_{2}$-induced oxidative stress triggers loss of mitofilin protein expression in the $\mathrm{ONH}$ astrocytes and whether $\mathrm{CoQ}_{10}$ treatment prevents this loss, we examined the protein expression level of mitofilin using western blot analysis. We found that oxidative stress triggered a significant loss of mitofilin protein expression by $0.36 \pm 0.04$-fold in the $\mathrm{ONH}$ astrocytes $(P<0.01$; Figure $4 \mathrm{c}$ ). In contrast, $\mathrm{CoQ}_{10}$ significantly promoted mitofilin protein expression by $0.59 \pm 0.02$-fold in the $\mathrm{ONH}$ astrocytes exposed to $\mathrm{H}_{2} \mathrm{O}_{2}$ compared with $\mathrm{H}_{2} \mathrm{O}_{2}$-treated $\mathrm{ONH}$ astrocytes $(P<0.05$; Figure 4c). However, there was no difference of porin protein expression between $\mathrm{H}_{2} \mathrm{O}_{2}$ and $\mathrm{CoQ}_{10} / \mathrm{H}_{2} \mathrm{O}_{2}$ treated $\mathrm{ONH}$ astrocytes (Figure $4 \mathrm{c}$ ), suggesting that $\mathrm{CoQ}_{10}$ could be protective in the mitochondria of $\mathrm{ONH}$ astrocytes by increasing the expression of mitofilin that in turn may protect mitochondrial cristae structure and ultimately OXPHOS capacity against oxidative stress. On the basis of increased mitochondrial mass by increasing mitofilin protein expression, we also determined whether $\mathrm{CoQ}_{10}$ triggers mitochondrial biogenesis using the antibody for peroxisome-proliferatoractivated receptor- $\gamma$ coactivator-1 (PGC-1 $1 \alpha$ ), a transcriptional coactivator and a mediator of mitochondrial biogenesis, in the $\mathrm{ONH}$ astrocytes exposed to $\mathrm{H}_{2} \mathrm{O}_{2}$. We found that oxidative stress significantly increased $\mathrm{PGC}-1 \alpha$ protein expression by $1.34 \pm 0.22$-fold in the $\mathrm{ONH}$ astrocytes 
a

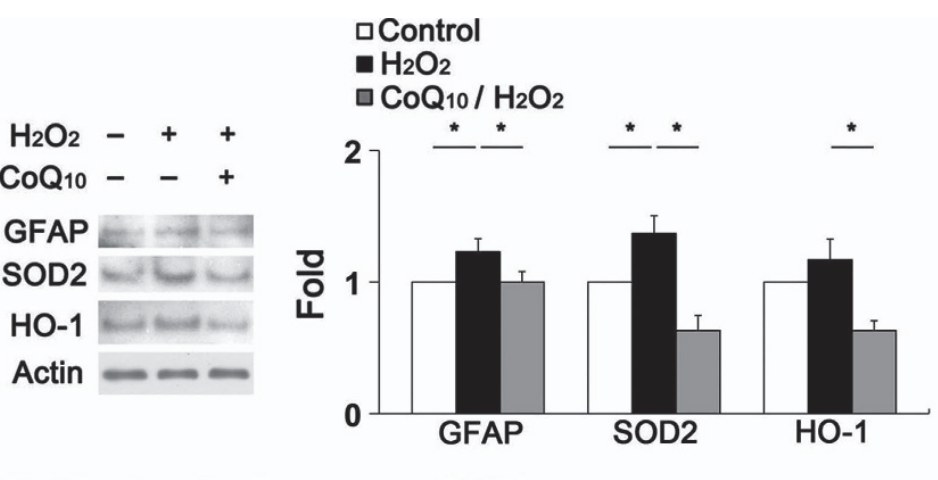

b MitoTracker Red
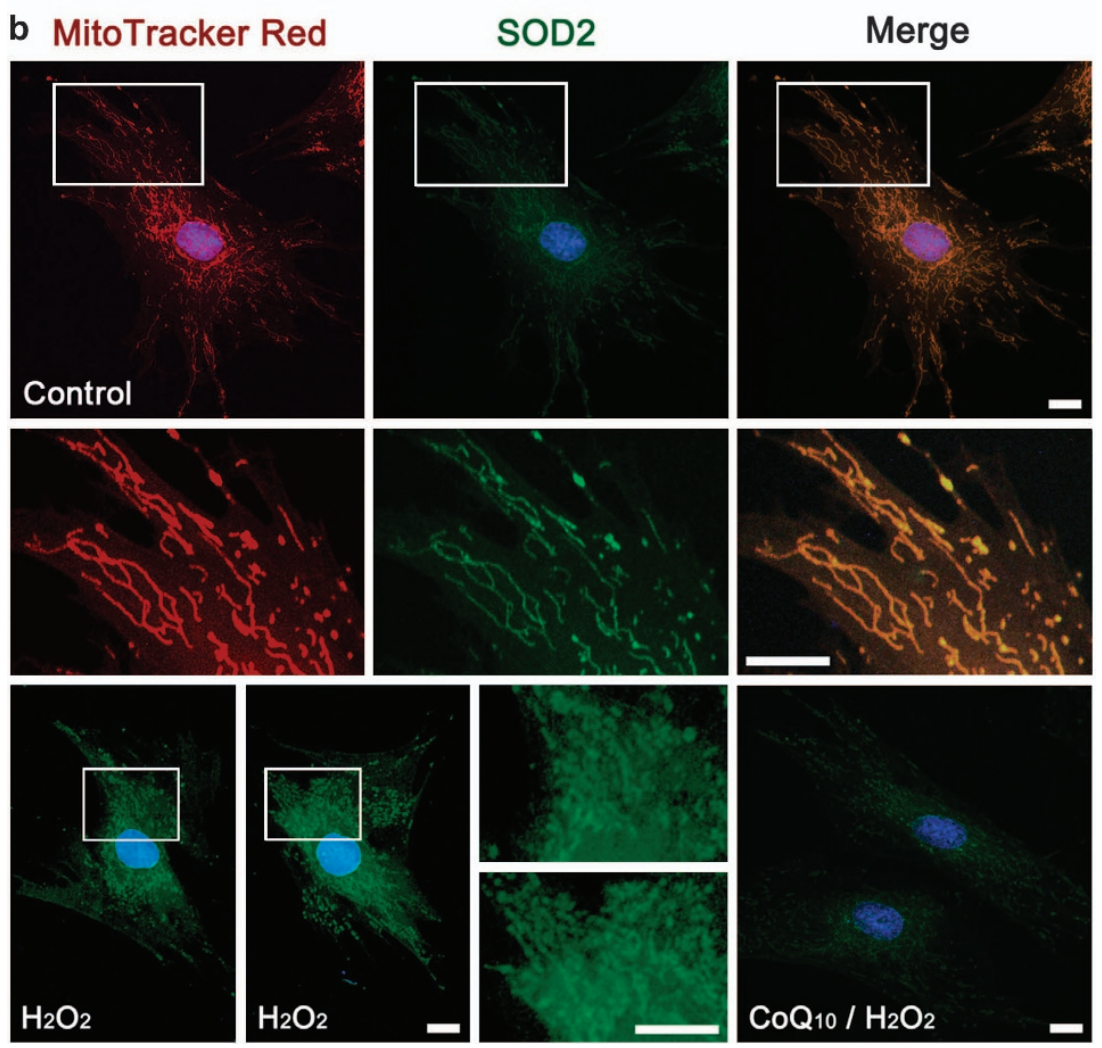

Figure $2 \mathrm{COQ}_{10}$ ameliorates oxidative stress in ONH astrocytes. (a) GFAP, SOD2 and HO-1 protein expression were significantly increased in the ONH astrocytes exposed to $\mathrm{H}_{2} \mathrm{O}_{2}(100 \mu \mathrm{M})$ compared with vehicle-treated control ONH astrocytes. In contrast, $\mathrm{CoQ}_{10}$ significantly reduced GFAP, SOD2 and HO-1 protein expression compared with $\mathrm{ONH}$ astrocytes exposed to $\mathrm{H}_{2} \mathrm{O}_{2}$. Relative intensity of chemiluminescence for each protein band was normalized using actin. Values are mean \pm S.D. ${ }^{*} \mathrm{P}<0.05$ compared with vehicle-treated control ONH astrocytes or $\mathrm{H}_{2} \mathrm{O}_{2}$-treated $\mathrm{ONH}$ astrocytes. (b) Representative images show that SOD2 immunoreactivity was present in mitochondria of the $\mathrm{ONH}$ astrocytes. Of note, SOD2 immunoreactivity was increased in the ONH astrocytes exposed to $\mathrm{H}_{2} \mathrm{O}_{2}$. However, CoQ $\mathrm{H}_{10}$ decreased SOD2 immunoreactivity in the $\mathrm{ONH}$ astrocytes exposed to $\mathrm{H}_{2} \mathrm{O}_{2}$. GFAP, glial fibrillary acidic protein; SOD2, superoxide dismutase 2; $\mathrm{HO}-1$, heme oxygenase-1; CoQ 10 , coenzyme Q10; $\mathrm{H}_{2} \mathrm{O}_{2}$, hydrogen peroxide. Scale bars, $10 \mu \mathrm{m}$

$\left(P<0.05 ;\right.$ Figure 4c). Of interest, $\mathrm{CoQ}_{10}$ showed greater increase of $\mathrm{PGC}-1 \alpha$ protein expression by $1.74 \pm 0.40$-fold in the $\mathrm{ONH}$ astrocytes exposed to $\mathrm{H}_{2} \mathrm{O}_{2}(P<0.05$; Figure $4 \mathrm{c})$, suggesting that $\mathrm{CoQ}_{10}$ could also be protective in the $\mathrm{ONH}$ astrocytes by triggering mitochondrial biogenesis against oxidative stress.

To better visualize the cristae, we performed electron tomography, a technique that provides the highest resolution three-dimensional (3D) structure determination of mitochondria. We generated tomographic reconstructions of 15 control, $60 \mathrm{H}_{2} \mathrm{O}_{2}$-exposed and $33 \mathrm{CoQ}_{10} / \mathrm{H}_{2} \mathrm{O}_{2}$-treated mitochondria and noticed that the cristae appeared dilated in the $\mathrm{H}_{2} \mathrm{O}_{2}$-exposed mitochondria (Figure 5). Measurements of cristae widths in the mitochondrial volumes showed that control cristae were $32 \pm 5 \mathrm{~nm}$ (mean \pm S.D.) across (membranes included), $\mathrm{CoQ}_{10} / \mathrm{H}_{2} \mathrm{O}_{2}$-treated cristae were $31 \pm 6 \mathrm{~nm}$ across, yet $\mathrm{H}_{2} \mathrm{O}_{2}$-exposed cristae were $48 \pm 17 \mathrm{~nm}$ across, a value statistically different from control and $\mathrm{CoQ}_{10} / \mathrm{H}_{2} \mathrm{O}_{2}$-treatment values $(P<0.001$, Figure 5$)$. The variation in cristae width was much greater in the $\mathrm{H}_{2} \mathrm{O}_{2}$-exposed mitochondria reflecting that not all the cristae were dilated. In comparison, the cristae widths in control and $\mathrm{CoQ}_{10} / \mathrm{H}_{2} \mathrm{O}_{2}$-treated mitochondria were more uniform (Figure 5). The abnormal cristae associated with oxidative stress are consistent with our finding of reduced ATP production. To further determine cristae abundance, we also 

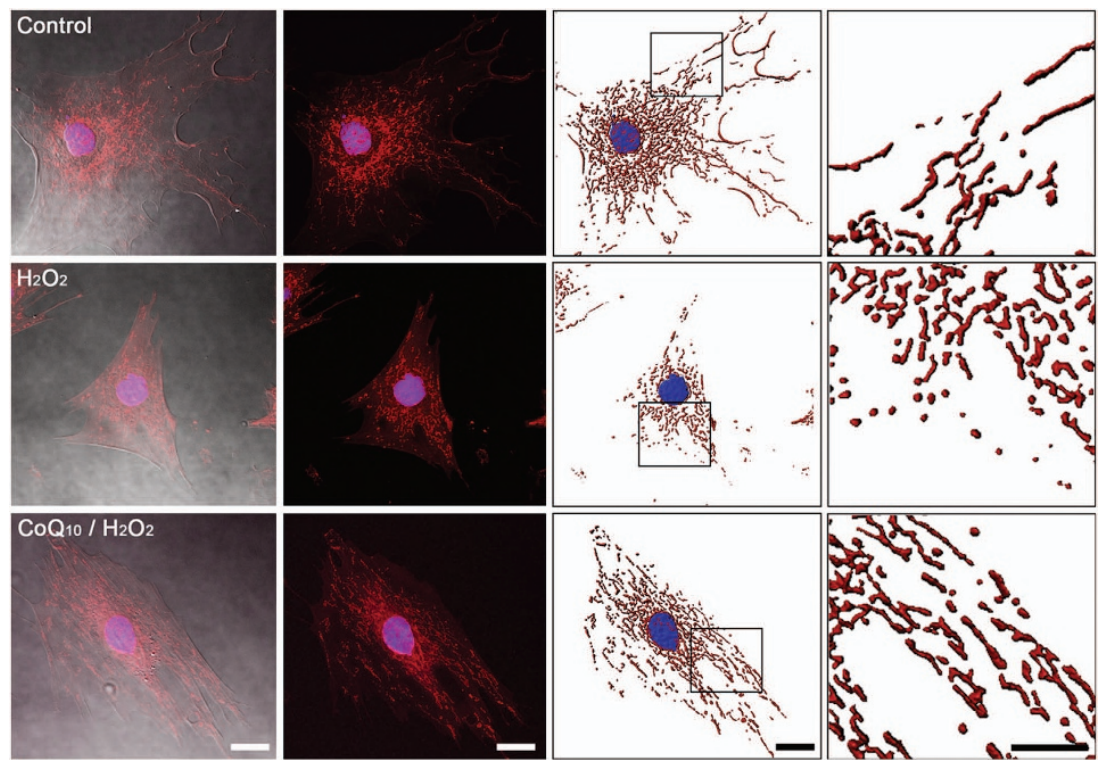

Figure $3 \mathrm{COQ}_{10}$ partially preserves mitochondrial structure of $\mathrm{ONH}$ astrocytes against oxidative stress-induced mitochondrial fission. The mitochondria of ONH astrocytes were stained with MitoTracker Red. Control ONH astrocytes exposed to vehicle contained classic elongated tubular mitochondria. In contrast, ONH astrocytes exposed to $\mathrm{H}_{2} \mathrm{O}_{2}$ contained small rounded mitochondria. However, $\mathrm{ONH}$ astrocytes pretreated with $\mathrm{CoQ}_{10}$ showed a partial preservation of mitochondrial morphology compared with the $\mathrm{ONH}$ astrocytes exposed to $\mathrm{H}_{2} \mathrm{O}_{2}$. $\mathrm{CoQ}_{10}$, coenzyme Q10; $\mathrm{H}_{2} \mathrm{O}_{2}$, hydrogen peroxide. Scale bars, $10 \mu \mathrm{m}$

performed measurment of mitochondrial cristae abundance. The parameter is cristae membrane surface area (SA) normalized to the outer membrane area, that is, cristae membrane SA divided by outer membrane SA that is dimensionless. Measurement of cristae abundance in the mitochondria showed that $\mathrm{H}_{2} \mathrm{O}_{2}$-exposed cristae significantly increased cristae abundance by $1.75 \pm 0.09(n=5$, mean \pm S.E.M.) compared with control cristae abundance by $0.95 \pm 0.26 \quad(n=5 ; P<0.05$, Figure 5). Pretreatment of $\mathrm{CoQ}_{10}$ decreased cristae abundance by $1.43 \pm 0.33(n=5)$ in the $\mathrm{ONH}$ astrocytes exposed to $\mathrm{H}_{2} \mathrm{O}_{2}$; however, there was no statistical difference between $\mathrm{H}_{2} \mathrm{O}_{2}$ and $\mathrm{CoQ}_{10} / \mathrm{H}_{2} \mathrm{O}_{2}$-treated $\mathrm{ONH}$ astrocytes (Figure 5).

$\mathrm{CoQ}_{10}$ preserves OXPHOS $\mathrm{Cx}$ protein expression against oxidative stress. Pretreatment of $\mathrm{CoQ}_{10}$ has been proposed to have a beneficial effect in the treatment of OXPHOS disorders. ${ }^{28-30}$ To determine whether $\mathrm{H}_{2} \mathrm{O}_{2}$-induced oxidative stress alters OXPHOS Cx protein expression and whether $\mathrm{CoQ}_{10}$ preserves OXPHOS $\mathrm{Cx}$ protein expression in $\mathrm{ONH}$ astrocytes, we examined the protein expression level of OXPHOS Cxs using western blot analysis. As shown in Figure 6, $\mathrm{H}_{2} \mathrm{O}_{2}$-induced oxidative stress significantly increased OXPHOS $\mathrm{Cx}$ (Cx I and II) protein expression by $2.56 \pm 0.08-(P<0.001)$ and $1.93 \pm 0.09$-fold $(P<0.01)$ compared with control $\mathrm{ONH}$ astrocytes, respectively. However, there were no significant difference in OXPHOS $\mathrm{Cx}(\mathrm{Cx} \mathrm{III}-\mathrm{V})$ protein expression between control and $\mathrm{H}_{2} \mathrm{O}_{2}$-treated $\mathrm{ONH}$ astrocytes. We also found that pretreatment of $\mathrm{CoQ}_{10}$ significantly decreased OXPHOS Cx (Cx I, II and V) protein expression by $0.33 \pm 0.06-(P<0.001), \quad 1.36 \pm 0.16-(P<0.01)$, and $0.62 \pm 0.07$-fold $(P<0.05)$, respectively, compared with $\mathrm{ONH}$ astrocytes exposed to $\mathrm{H}_{2} \mathrm{O}_{2}$, However, there was no significant difference in OXPHOS Cx (Cx III and IV) protein expression between $\mathrm{H}_{2} \mathrm{O}_{2}$ - and $\mathrm{CoQ}_{10} / \mathrm{H}_{2} \mathrm{O}_{2}$-treated $\mathrm{ONH}$ astrocytes, indicating that $\mathrm{CoQ}_{10}$ may preserve OXPHOS Cx protein expression in the $\mathrm{ONH}$ astrocytes against oxidative stress.

\section{$\mathrm{CoQ}_{10}$ prevents alteration of mitochondrial bioenergetics} due to oxidative stress. To determine whether $\mathrm{H}_{2} \mathrm{O}_{2}$-induced oxidative stress alters mitochondrial bioenergetics in $\mathrm{ONH}$ astrocytes and whether $\mathrm{CoQ}_{10}$ treatment inhibits the alteration of mitochondrial bioenergetics, we measured cell viability, ROS generation and cellular ATP level. As shown in Figure 7, our findings show that $\mathrm{H}_{2} \mathrm{O}_{2}$-induced oxidative stress significantly decreased cell viability to $80.8 \pm 5.4 \%$ of the control value in the $\mathrm{ONH}$ astrocytes $(P<0.05)$. However, pretreatment of $\mathrm{CoQ}_{10}$ significantly boosted cell viability to $92.6 \pm 4.6 \%$ in the $\mathrm{ONH}$ astrocytes exposed to $\mathrm{H}_{2} \mathrm{O}_{2}(P=0.001)$. In comparison with control $\mathrm{ONH}$ astrocytes, $\mathrm{H}_{2} \mathrm{O}_{2}$-induced oxidative stress significantly increased ROS generation by $7.82 \pm 0.81$-fold over the control in the $\mathrm{ONH}$ astrocytes $(P=0.01)$. In contrast, pretreatment of $\mathrm{CoQ}_{10}$ significantly decreased $\mathrm{ROS}$ generation to $4.67 \pm 0.83$-fold of the control in the $\mathrm{ONH}$ astrocytes exposed to $\mathrm{H}_{2} \mathrm{O}_{2} \quad(P<0.05)$. In addition, we found that $\mathrm{H}_{2} \mathrm{O}_{2}$-induced oxidative stress significantly decreased the cellular ATP level to $83.8 \pm 3.7 \%$ of the control value in the $\mathrm{ONH}$ astrocytes $(P<0.01)$. However, pretreatment of $\mathrm{CoQ}_{10}$ nearly completely restored the cellular ATP level $(96.9 \pm 4.3 \%$ of the control) in the $\mathrm{ONH}$ astrocytes exposed to $\mathrm{H}_{2} \mathrm{O}_{2}$ $(P<0.05)$, suggesting that $\mathrm{CoQ}_{10}$ restores bioenergetic function in the $\mathrm{ONH}$ astrocytes to counter oxidative stress.

\section{Discussion}

We addressed the question of whether oxidative stress triggers mitochondria-mediated dysfunction in the $\mathrm{ONH}$ 
astrocytes because oxidative stress has been implicated as an important pathophysiological mechanisms in the pathogenesis of glaucoma. ${ }^{4,9,31,32}$ It has been proposed that reduction of oxidative stress could be protective in glaucomatous $\mathrm{ONH}$ treatment ${ }^{24,33-35}$ and that mitochondrial dysfunction may have a critical role in oxidative stress-mediated glaucomatous

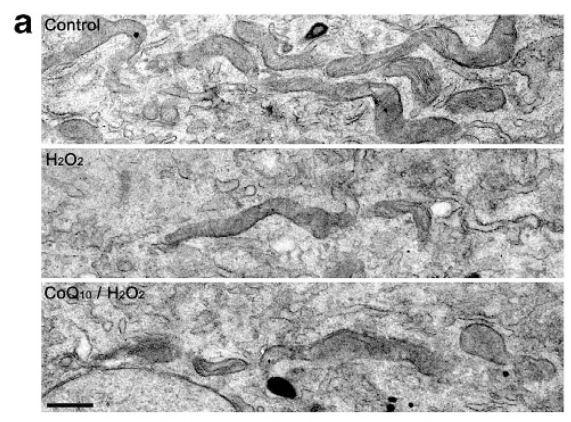

b
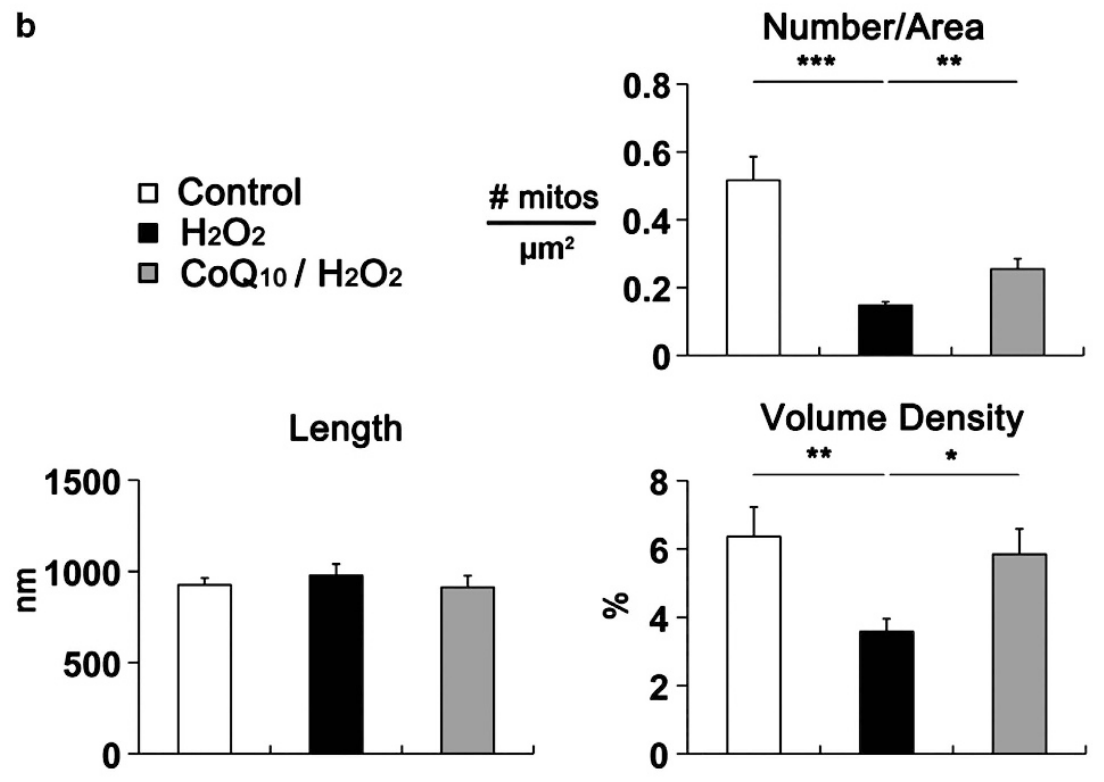

c
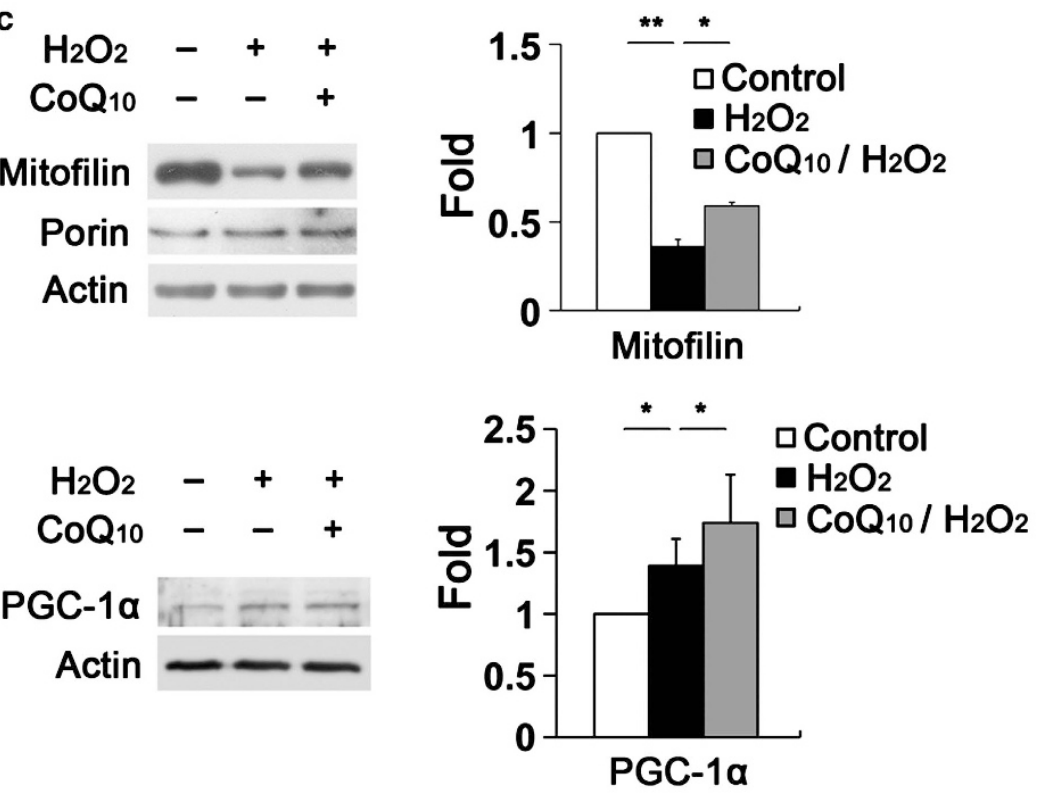
neurodegeneration. ${ }^{2,4,5,36,37}$ Interestingly, oxidative stress leads to antioxidant or immune responses in human $\mathrm{ONH}$ astrocytes. ${ }^{24,32,38}$ Support for our investigations came from a recent study demonstrating oxidative stress, mitochondrial dysfunction and $\mathrm{Ca}^{2+}$ overload in GFAP-negative human lamina cribrosa cells in the $\mathrm{ONH}$ from glaucoma donor eyes. ${ }^{3}$
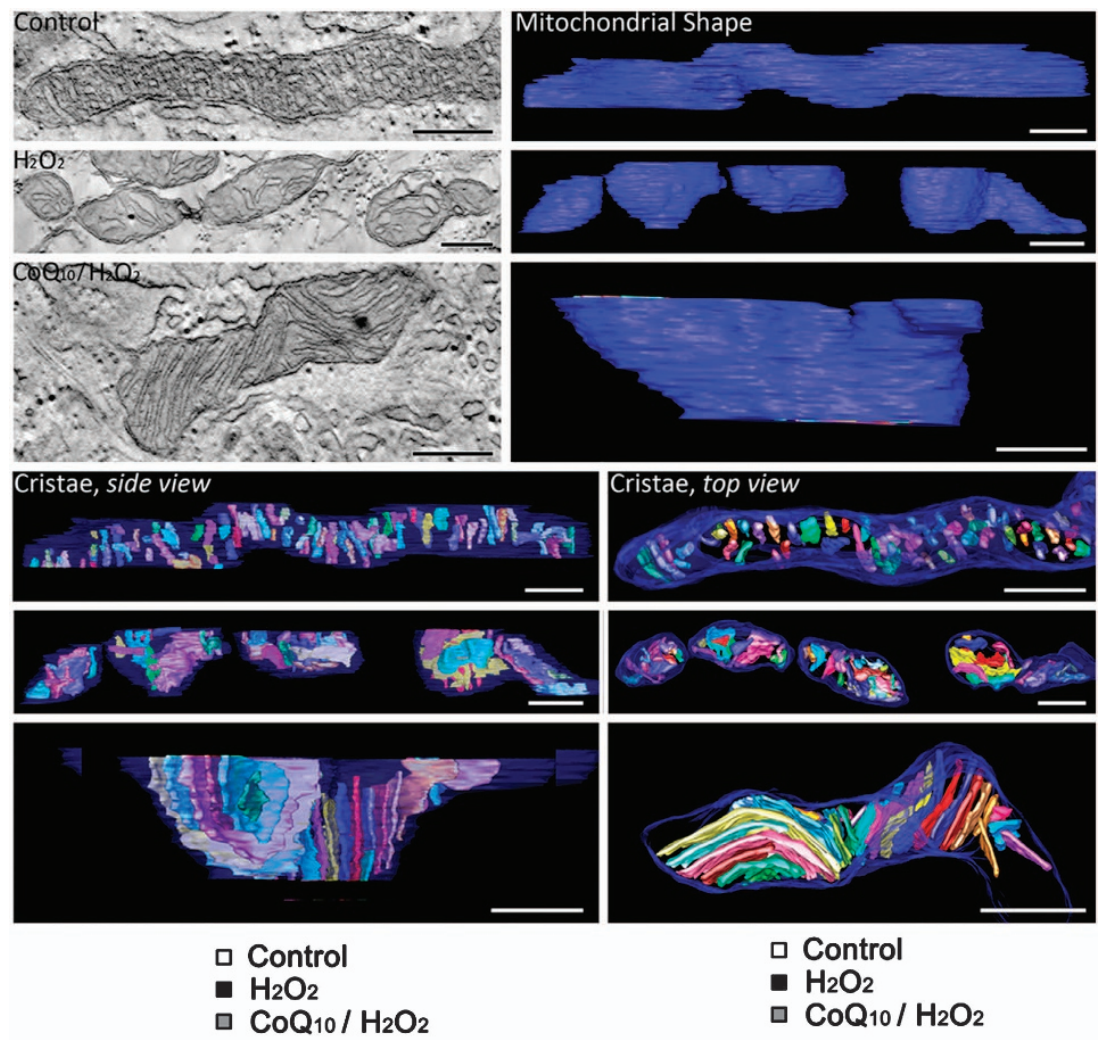
$\square$ Control
- $\mathrm{H}_{2} \mathrm{O}_{2}$
$\mathrm{CoQ}_{10} / \mathrm{H}_{2} \mathrm{O}_{2}$
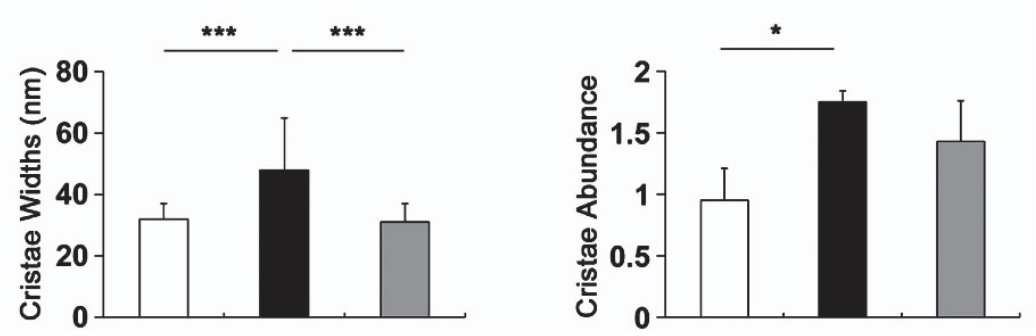

Figure $53 \mathrm{D}$ reconstruction of mitochondrial cristae in the $\mathrm{ONH}$ astrocytes. Oxidative stress dilated cristae that were prevented by $\mathrm{CoQ}_{10} / \mathrm{H}_{2} \mathrm{O}_{2}$ treatment. Electron tomography generated high-resolution, 3D reconstructions of control, $\mathrm{H}_{2} \mathrm{O}_{2}$-exposed and $\mathrm{CoQ}_{10} / \mathrm{H}_{2} \mathrm{O}_{2}$-treated mitochondria. Slices (1.4-nm thick) through the middle of electron microscopy tomographic volumes of mitochondria are shown on the left. Surface-rendered volumes of the segmented mitochondria provide information concerning shape and cristae architecture. The outer mitochondrial membrane is shown in blue (made translucent to better visualize the cristae) and cristae are in various colors. The long control mitochondrion has 46 cristae, the $\mathrm{H}_{2} \mathrm{O}_{2}$-exposed has 47 cristae distributed in the four mitochondria that are lined up and the $\mathrm{CoQ}_{10} / \mathrm{H}_{2} \mathrm{O}_{2}$-treated mitochondrion has 27 cristae. The mean of cristae widths is $50 \%$ greater in the $\mathrm{H}_{2} \mathrm{O}_{2}$-exposed mitochondria compared with the control and $\mathrm{CoQ}_{10}$ pretreatment samples. Scale bar, $250 \mathrm{~nm}$ (all

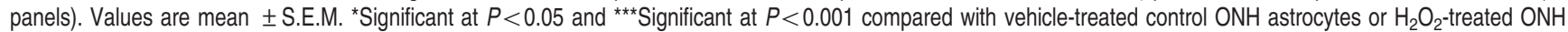
astrocytes. Representative graphs show the measurement of cristae widths and abundance in the mitochondria. $\mathrm{CoQ}_{10}$, coenzyme $\mathrm{Q} 10 ; \mathrm{H}_{2} \mathrm{O}_{2}$, hydrogen peroxide

Figure $4 \mathrm{CoQ}_{10}$ triggers mitochondrial biogenesis in $\mathrm{ONH}$ astrocytes that counters oxidative stress. (a) The representative $2 \mathrm{D}$ images from TEM analysis showed that control ONH astrocytes exposed to vehicle contained classic elongated tubular mitochondria. However, ONH astrocytes exposed to $\mathrm{H}_{2} \mathrm{O}_{2}$ contained fewer mitochondria in the $\mathrm{ONH}$ astrocytes. Interestingly, $\mathrm{ONH}$ astrocytes pretreated with $\mathrm{CoQ}_{10}$ showed a greater number compared with the $\mathrm{ONH}$ astrocytes exposed to $\mathrm{H}_{2} \mathrm{O}_{2}$. (b) Quantitative analysis showed that mitochondrial number per area and volume density were significantly decreased in the $\mathrm{ONH}$ astrocytes exposed to $\mathrm{H}_{2} \mathrm{O}_{2}$. However, ONH astrocytes pretreated with $\mathrm{CoQ}_{10}$ had a significant increase in mitochondrial number and volume density compared with the $\mathrm{ONH}$ astrocytes exposed to $\mathrm{H}_{2} \mathrm{O}_{2}$. There was no difference in mitochondrial length among control, $\mathrm{H}_{2} \mathrm{O}_{2}$ and $\mathrm{CoQ}_{10} / \mathrm{H}_{2} \mathrm{O}_{2}$-treated $\mathrm{ONH}$ astrocytes. Values are mean \pm S.E.M. ${ }^{*} P<0.05,{ }^{* *} P<0.01$ and ${ }^{* \star *} P<0.001$ compared with vehicle-treated control $\mathrm{ONH}$ astrocytes or $\mathrm{H}_{2} \mathrm{O}_{2}$-treated $\mathrm{ONH}$ astrocytes. Scale bar, $500 \mathrm{~nm}$. (c) Mitofilin protein expression was significantly increased in the $\mathrm{ONH}$ astrocytes exposed to $\mathrm{H}_{2} \mathrm{O}_{2}$ compared with vehicle-treated control $\mathrm{ONH}$ astrocytes. In contrast, $\mathrm{COQ}_{10}$ significantly increased mitofilin protein expression compared with $\mathrm{ONH}$ astrocytes exposed to $\mathrm{H}_{2} \mathrm{O}_{2}$. PGC- $1 \alpha$ protein expression was significantly increased in the $\mathrm{ONH}$ astrocytes exposed to $\mathrm{H}_{2} \mathrm{O}_{2}$ compared with vehicle-treated control ONH astrocytes. Of interest, pretreatment of $\mathrm{CoQ}_{10}$ showed greater increase of $\mathrm{PGC}-1 \alpha$ protein expression in the $\mathrm{ONH}$ astrocytes exposed to $\mathrm{H}_{2} \mathrm{O}_{2}$. Relative intensity of chemiluminescence for each protein band was normalized using actin. Values are mean \pm S.D. $(n=3) . \mathrm{CoQ}_{10}$, coenzyme $\mathrm{Q} 10 ; \mathrm{H}_{2} \mathrm{O}_{2}$, hydrogen peroxide 

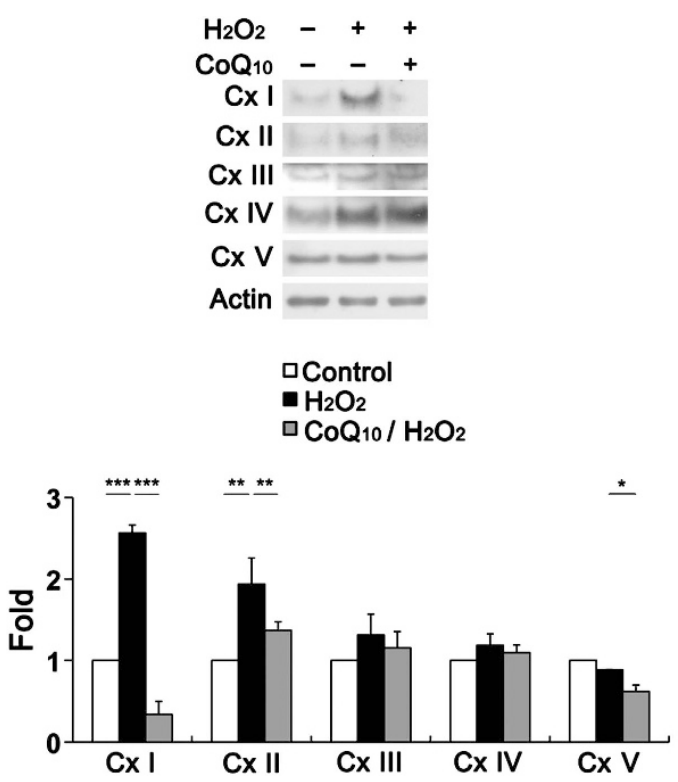

Figure $6 \mathrm{COQ}_{10}$ preserves OXPHOS Cx protein expression against oxidative stress. In comparison with control $\mathrm{ONH}$ astrocytes, $\mathrm{H}_{2} \mathrm{O}_{2}$-induced oxidative stress significantly increased OXPHOS $\mathrm{Cx}(\mathrm{Cx} I$ and II) protein expression in the $\mathrm{ONH}$ astrocytes. However, there was no significant difference in OXPHOS CX (Cx III-V) protein expression. Pretreatment of $\mathrm{CoQ}_{10}$ significantly decreased OXPHOS Cx (Cx I, II and V) protein expression compared with $\mathrm{ONH}$ astrocytes exposed to $\mathrm{H}_{2} \mathrm{O}_{2}$. However, there was no significant difference in OXPHOS Cx (Cx III and IV) protein expression between $\mathrm{H}_{2} \mathrm{O}_{2}$ - and $\mathrm{CoQ}_{10} / \mathrm{H}_{2} \mathrm{O}_{2}$-treated $\mathrm{ONH}$ astrocytes. Values are mean \pm S.D. ${ }^{*} P<0.05,{ }^{* *} P<0.01$ and ${ }^{* * *} P<0.001$ compared with vehicle-treated control $\mathrm{ONH}$ astrocytes or $\mathrm{H}_{2} \mathrm{O}_{2}$-treated $\mathrm{ONH}$ astrocytes. $\mathrm{CoQ}_{10}$, coenzyme Q10; $\mathrm{H}_{2} \mathrm{O}_{2}$, hydrogen peroxide; OXPHOS, oxidative phosphorylation; $\mathrm{Cx}$, complex

$\mathrm{CoQ}_{10}$ is an attractive antioxidant and neurotherapeutic agent for us to test efficacy in glaucoma because published evidence supports its effectiveness against oxidative stress in many neurodegenerative diseases including Parkinson' and Huntington' diseases. ${ }^{15,39,40}$ Recent studies increasingly demonstrated that $\mathrm{CoQ}_{10}$ is neuroprotective in retinal cells in vivo and in vitro against pressure, oxidative stress, excitotoxicity, or apoptotic radiation. ${ }^{19,21,22,41,42}$ In the current study, we found that oxidative stress triggers the activation of $\mathrm{ONH}$ astrocytes as well as the upregulation of SOD2 and $\mathrm{HO}-1$ protein expression in the $\mathrm{ONH}$ astrocytes. In contrast, $\mathrm{CoQ}_{10}$ not only prevents activation of $\mathrm{ONH}$ astrocytes but also significantly decreases SOD2 and $\mathrm{HO}-1$ protein expression in the $\mathrm{ONH}$ astrocytes against oxidative stress. The upregulation of antioxidative enzymes such as SOD2 in astrocytes is accompanied by a higher resistance to oxidative stress induced by $\mathrm{H}_{2} \mathrm{O}_{2}{ }^{43}$ In addition, SOD2 activity directly stabilizes mitochondrial transmembrane potential and calcium-buffering ability. ${ }^{44}$ Together with these findings, our results, therefore, raise the possibility that increasing SOD2 or $\mathrm{HO}-1$ expression in the $\mathrm{ONH}$ astrocytes may contribute to compensatory endogenous antioxidant mechanisms that increase resistance or stabilization of mitochondria against oxidative stress. In addition, these results reflect that $\mathrm{CoQ}_{10}$ could be an important antioxidant for ameliorating oxidative stress-mediated dysfunction in the $\mathrm{ONH}$ astrocyte. We believe that studying the benefits of $\mathrm{CoQ}_{10}$-mediated blockade of oxidative stress or enhancement of antioxidant enzymes in the $\mathrm{ONH}$ astrocytes may be rewarding in many optic neuropathy including glaucoma.

We further addressed the relationship between oxidative stress and the mitochondrial network in the $\mathrm{ONH}$ astrocytes. We demonstrated for the first time that oxidative stress induced a significant loss of mitochondrial mass by decreasing mitochondrial number and volume density in the $\mathrm{ONH}$ astrocytes. However, $\mathrm{CoQ}_{10}$ treatment showed a partial inhibition of mitochondrial loss by increasing mitochondrial number and volume density in the $\mathrm{ONH}$ astrocytes, suggesting that $\mathrm{CoQ}_{10}$ could protect $\mathrm{ONH}$ astrocytes by triggering mitochondrial biogenesis. In agreement with these findings, we also observed that oxidative stress significantly reduced the expression level of mitofilin protein in the $\mathrm{ONH}$ astrocytes. However, there was no significant difference in porin expression among groups, suggesting that the observed increase in number of mitochondria did not produce significantly more outer membrane where porin resides or alternatively that porin was diluted upon mitochondrial biogenesis. Intriguingly, $\mathrm{CoQ}_{10}$ treatment partially increased mitofilin protein expression in the $\mathrm{ONH}$ astrocytes against oxidative stress. More importantly, we also found that $\mathrm{CoQ}_{10}$ treatment showed greater increase of PGC- $1 \alpha$ protein expression in the $\mathrm{ONH}$ astrocytes to protect against oxidative stress.

Mitofilin is anchored to the mitochondrial inner membrane and controls mitochondrial cristae morphology. ${ }^{26}$ As a previous study suggests that mitofilin depletion decreases cellular proliferation and increases apoptosis, as well as triggers mitochondrial structural and functional abnormalities, ${ }^{26}$ it is possible that a significant loss of mitofilin protein expression by oxidative stress may result in structural and functional dysfunction of mitochondria in the $\mathrm{ONH}$ astrocytes. PGC- $1 \alpha$ activates mitochondrial biogenesis and oxidative metabolism ${ }^{45,46}$ as well as regulates the transcription target proteins such as nuclear-encoded respiratory $\mathrm{Cx}$ proteins and mitochondrial transcription factor $A$ that has an important role in mitochondrial gene expression and mitochondrial DNA maintenance, and therefore is essential for OXPHOSmediated ATP synthesis. ${ }^{45,47,48}$ Collectively, therefore, increasing mitofilin or PGC-1 $\alpha$ protein expression by $\mathrm{CoQ}_{10}$ treatment also raises the intriguing possibility that mitofilin or PGC- $1 \alpha$ can be a therapeutic target for protecting mitochondria or promoting mitochondrial biogenesis, especially cristae biogenesis or stablization of the $\mathrm{ONH}$ astrocytes to counter oxidative stress-mediated mitochondrial dysfunction. In addition, we found that oxidative stress significantly increased cristae widths and abundance in the mitochondria of the $\mathrm{ONH}$ astocytes. However, $\mathrm{CoQ}_{10}$ treatment significantly preserved cristae widths, but partially preserved cristae abundance in the mitochondria of the $\mathrm{ONH}$ astrocytes against oxidative stress, suggesting that $\mathrm{CoQ}_{10}$ can modulate mitochondrial cristae structure in the $\mathrm{ONH}$ astrocytes to protect against oxidative damage. Altogether, our findings suggest that $\mathrm{CoQ}_{10}$ may have a therapeutic potential for ameliorating oxidative stress-induced alteration of the mitochondrial network in the $\mathrm{ONH}$ astrocytes. Future studies are needed to address how $\mathrm{CoQ}_{10}$ directly promotes mitochondrial biogenesis and structural preservation in the $\mathrm{ONH}$ astrocytes against oxidative stress and whether oxidative stress-mediated mitofilin loss directly alters mitochondrial cristae morphology. 

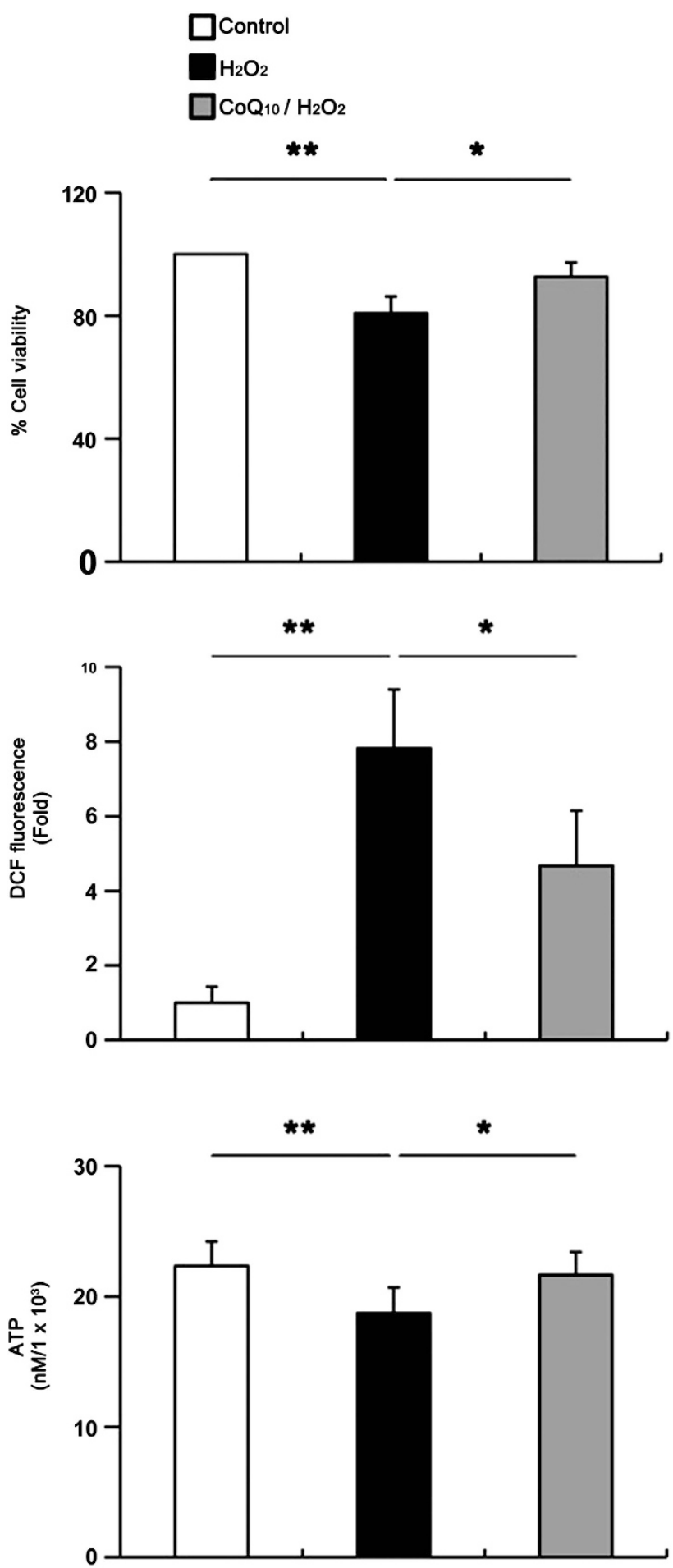

Figure $7 \quad \mathrm{Co}_{10}$ prevents the alteration of mitochondrial bioenergetics caused by oxidative stress. Pretreatment of $\mathrm{CoQ}_{10}$ significantly restored cell viability to $92.6 \pm 4.6 \%$ of the control level in the $\mathrm{ONH}$ astrocytes exposed to $\mathrm{H}_{2} \mathrm{O}_{2}$. In comparison with control $\mathrm{ONH}$ astrocytes, $\mathrm{H}_{2} \mathrm{O}_{2}$-induced oxidative stress significantly increased ROS generation in the $\mathrm{ONH}$ astrocytes. In contrast, pretreatment of $\mathrm{CoQ}_{10}$ significantly decreased ROS generation in the ONH astrocytes exposed to $\mathrm{H}_{2} \mathrm{O}_{2}$. In addition, $\mathrm{H}_{2} \mathrm{O}_{2}$-induced oxidative stress significantly decreased the cellular ATP level in the $\mathrm{ONH}$ astrocytes. However, pretreatment of $\mathrm{Co}_{10}$ significantly restored the cellular ATP level in the ONH astrocytes exposed to $\mathrm{H}_{2} \mathrm{O}_{2}$. Values are mean \pm S.D. ${ }^{*} P<0.05$ and ${ }^{* *} P<0.01$ compared with vehicle-treated control ONH astrocytes or $\mathrm{H}_{2} \mathrm{O}_{2}$-treated $\mathrm{ONH}$ astrocytes. $\mathrm{CoQ}_{10}$, coenzyme Q10; $\mathrm{H}_{2} \mathrm{O}_{2}$, hydrogen peroxide
In the current study, we found that oxidative stress triggered the upregulation of OXPHOS CXS (Cx I-IV) protein expression in the $\mathrm{ONH}$ astocytes. However, $\mathrm{CoQ}_{10}$ treatment preserved OXPHOS Cx protein expression including the ATP synthase against oxidative stress. $\mathrm{CoQ}_{10}$ regulates the mitochondrial OXPHOS system and prevents lipid peroxidation. ${ }^{49,50}$ It has been demonstrated that a defective OXPHOS system may be induced by an insufficient fuel supply, defective electron transport chain enzymes (Cx I-IV) or lack of the electron carrier $\mathrm{CoQ}_{10}{ }^{49}$ In addition, $\mathrm{CoQ}_{10}$ treatment significantly increases ATP synthetic capacity in lymphocytes from patients as well as in cultured control lymphocytes in vitro, ${ }^{28}$ suggesting that $\mathrm{CoQ}_{10}$ may have a beneficial effect in the treatment of OXPHOS disorders. ${ }^{28,29}$ Although further investigations are needed, it is possible that oxidative stress may induce $\mathrm{CoQ}_{10}$ deficiency in $\mathrm{ONH}$ astrocytes, triggering alteration of the OXPHOS system. Further, our findings showed that $\mathrm{CoQ}_{10}$-mediated partial preservation of mitochondrial cristae abundance supports $\mathrm{CoQ}_{10}$-mediated increase of certain OXPHOS Cxs against oxidative stress. Thus, our results reflect that the upregulation of OXPHOS Cx protein expression may contribute to a critical endogenous defense mechanism for protecting mitochondria of the $\mathrm{ONH}$ astrocytes against oxidative stress.

Oxidative stress-mediated ROS alters OXPHOS function. ${ }^{51,52}$ This alteration of mitochondrial OXPHOS Cxs by oxidative stress in turn leads to reduction of ATP production and increase of ROS generation, consistent with our results. ${ }^{2}$ Further, increased ROS generation results in mitochondrial bioenergetic dysfunction in many neurodegenerative diseases, ${ }^{53,54}$ suggesting an oxidative stress-mediated mitochondrial vicious cycle involved in neurodegeneration that includes oxidative stress, ROS production, OXPHOS alteration and mitochondrial bioenergetic dysfunction. In the current study, we found that oxidative stress triggers an excessive increase of ROS generation, reduction of cellular ATP level and subsequent decrease of cell viability in the $\mathrm{ONH}$ astrocytes. Of note, we found that $\mathrm{CoQ}_{10}$ treatment decreases ROS generation, increases cellular ATP production, and promotes cell viability in $\mathrm{ONH}$ astrocytes. It was shown that $\mathrm{CoQ}_{10}$ improves mitochondrial bioenergetic parameters such as oxygen consumption rate, mitochondrial transmembrane potential and ATP synthesis in cultured T62 and $\mathrm{H} 9 \mathrm{c} 2$ cell lines consistent with its role in the mitochondrial respiratory chain. ${ }^{55}$ Moreover, a recent study demonstrated that $\mathrm{CoQ}_{10}$ deficiency is associated with oxidative stress, increased ROS production, defective ATP synthesis and cell death. $^{56}$ Our findings also showed that oxidative stress triggered abnormal cristae morphology such as cristae dilation in the mitochondria of the $\mathrm{ONH}$ astroctyes. Moreover, because activation of PGC- $1 \alpha$ correlates with increased cellular energy demand, ${ }^{57}$ a significant increase of PGC- $1 \alpha$ may reflect an increased energy demand from the mitochondria of $\mathrm{ONH}$ astrocytes against oxidative stress. This correlates with our findings of reduced ATP production. Altogether, these findings strongly suggest that $\mathrm{CoQ}_{10}$ supplementation may have therapeutic potential for ameliorating a mitochondrial vicious cycle involved in $\mathrm{ONH}$ degeneration that includes oxidative stress, OXPHOS dysfunction, ROS generation and bioenergetics impairment in the $\mathrm{ONH}$ 
astrocytes during glaucomatous neurodegeneration. Finally, we believe that it would be useful to measure the origin of the energy defect by pharmacologically inhibiting glycolytic and mitochondrial pathways in the future studies.

On the basis of these observations, we suggest that oxidative stress-mediated mitochondrial dysfunction or alteration may be important pathophysiological mechanisms in the dysfunction of $\mathrm{ONH}$ astrocytes. Therefore, treatment with $\mathrm{CoQ}_{10}$ may be neuroprotective and protect $\mathrm{ONH}$ astrocytes against oxidative stress-mediated mitochondrial dysfunction or alteration in glaucoma and other optic neuropathies.

\section{Materials and Methods}

Animals. Pregnant Sprague-Dawley rats $(250-300 \mathrm{~g}$ in weight; Harlan Laboratories, Indianapolis, IN, USA) were housed in covered cages, fed with a standard rodent diet ad libitum, and kept on a 12-h light/12-h dark cycle. All procedures concerning animals were in accordance with the ARVO Statement for the Use of Animals in Ophthalmic Vision Research and under protocols approved by institutional IACUC committees at the University of California, San Diego.

Isolation and culture of rat $\mathrm{ONH}$ astrocytes. Primary rat $\mathrm{ONH}$ astrocyte cultures were modified and derived as previously described. ${ }^{23}$ After euthanizing with $\mathrm{CO}_{2}$ gas, 10-20 pieces of $\mathrm{ONH}$ tissue were dissected from postnatal day 5 Sprague-Dawley rats and transferred to a $35-\mathrm{mm}$ petri dish with $2 \mathrm{ml} 0.2 \%$ bovine serum albumin (BSA)/Dulbecco's phosphate-buffered saline (DPBS). Under a dissecting microscope, pigmented and remnant non-neuronal tissues such as sclera and other neighboring tissues were removed, and the $\mathrm{ONH}$ tissue was identified and dissected using a microscissor and sharp blade. The $\mathrm{ONH}$ tissue was minced and transferred to a $60-\mathrm{mm}$ petri dish and conditioned with growth medium: GlutaMAX/Dulbecco's modified Eagle's medium (DMEM) supplemented with $10 \%$ fetal bovine serum (FBS), $100 \mathrm{U} / \mathrm{ml}$ penicillin and $100 \mu \mathrm{g} / \mathrm{ml}$ streptomycin (Invitrogen, La Jolla, CA, USA), and prewarmed in a humidified atmosphere $5 \% \mathrm{CO}_{2}$ incubator at $37^{\circ} \mathrm{C}$. After incubation for 10 days, the $\mathrm{ONH}$ explants were removed by $70-\mu \mathrm{m}$ cell strainers (BD, Sparks, MD, USA). The cells that were grown from the $\mathrm{ONH}$ explants were plated in a $100-\mathrm{mm}$ petri dish and incubated for 3 days at $37^{\circ} \mathrm{C}$. For further purification of the $\mathrm{ONH}$ astrocytes, the growth medium was changed to serum-deprived medium and the culture dish was shaken for $24 \mathrm{~h}$ at room temperature. After removing nonadherent cells, the adherent $\mathrm{ONH}$ astrocytes were collected, centrifuged and replated on poly-L-lysine-coated culture dishes. The homogeneity of ONH astrocyte cultures was evaluated by western blot analysis or immunohistochemistry as below.

Pharmacological treatment. Three groups of rat $\mathrm{ONH}$ astrocytes were studied following treatment of $\mathrm{H}_{2} \mathrm{O}_{2}$ or $\mathrm{CoQ}_{10}$ : a group treated with vehicle (chloroform; Sigma, St. Louis, MO, USA), a group treated with $100 \mu \mathrm{M} \mathrm{H}_{2} \mathrm{O}_{2}$ (Sigma), and a group treated with $\mathrm{CoQ}_{10}(50 \mu \mathrm{g} / \mathrm{ml}$ in chloroform, Sigma) and $100 \mu \mathrm{M} \mathrm{H}_{2} \mathrm{O}_{2}$ (Sigma). $\mathrm{H}_{2} \mathrm{O}_{2}$ was treated in $1 \% \mathrm{FBS} / \mathrm{DMEM} /$ Ham's $\mathrm{F} 12$ 50/50 (DMEM/F12) for $1 \mathrm{~h}$ in a $5 \% \mathrm{CO}_{2}$ incubator at $37^{\circ} \mathrm{C}$. $\mathrm{CoQ}_{10}$ was pre-incubated with $1 \% \mathrm{FBS} / \mathrm{DMEM} / \mathrm{F} 12$ for $24 \mathrm{~h}$ and treated with $\mathrm{H}_{2} \mathrm{O}_{2}$ with $\mathrm{DMEM} / \mathrm{F} 12$ for $1 \mathrm{~h}$ in a $5 \% \mathrm{CO}_{2}$ incubator at $37^{\circ} \mathrm{C}$.

Western blot analysis. Rat $\mathrm{ONH}$ astrocytes were lysed with lysis buffer (20 mM HEPES pH 7.0, 1\% TritonX-100, 10\% glycerol, 2 mM EGTA, 1 mM EDTA $\mathrm{pH} 8.0$ (w/ $/ \mathrm{NaOH}), 20 \mathrm{mM} \beta$-glycerolphosphate, complete protease inhibitors (Roche Biochemicals, Indianapolis, IN, USA) and phosphatase inhibitors $(1 \mu \mathrm{g} / \mathrm{ml}$ Aprotinin, $1 \mu \mathrm{g} / \mathrm{ml}$ Leupeptin, $1 \mathrm{mM}$ AEBSF, $1 \mathrm{mM} \mathrm{Na}_{3} \mathrm{VO}_{4}, 5 \mathrm{mM} \mathrm{NaF}$ and $2.5 \mathrm{mM}$ Microcystin-LR)). Each sample (10 mg proteins per well) was loaded and separated based on the molecular weights by SDS-PAGE and electrotransferred to polyvinylidene difluoride membranes. The membranes were blocked with $5 \%$ nonfat dry milk with $0.1 \%$ Tween-20 in PBS (PBST) for $1 \mathrm{~h}$ at room temperature and incubated with primary antibodies at $4{ }^{\circ} \mathrm{C}$ for overnight. The primary antibodies used were mouse monoclonal anti-GFAP antibody (1:3000; Sigma), rabbit polyclonal anti-lba1 antibody (1:500; Wako Chemicals, Richmond, VA, USA), rabbit polyclonal anti-MBP antibody (1:1000; Abcam, Cambridge, MA, USA), rabbit polyclonal anti-SOD2 antibody (1:2000; Santa Cruz Biotechnology, Santa Cruz, CA, USA), rabbit polyclonal anti-HO-1 antibody (1:5000; Stressgen
Bioreagents, San Diego, CA, USA), mouse monoclonal anti-mitofilin antibody (1: 500; Mitosciences, Eugene, OR, USA), rabbit polyclonal anti-PGC-1 $\alpha$ (1:1000; Santa Cruz Biotechnology), mouse monoclonal anti-total OXPHOS Cx antibody (containing a mixture of antibodies to CXI-IV and ATP synthase, 1:3000; Invitrogen), rabbit polyclonal anti-porin antibody (1:2000; Calbiochem, Rockland, MA, USA) and mouse monoclonal anti-actin antibody (1:5000; Millipore, Billerica, MA, USA). After several washes with PBST, the membranes were incubated for $1 \mathrm{~h}$ at room temperature with horseradish peroxidase-conjugated goat anti-mouse IgG (1:5000; Bio-Rad, Hercules, CA, USA) or goat anti-rabbit IgG (1:5000; Bio-Rad) and developed by chemiluminescence detection. The density of protein bands from the scanned images was analyzed using ImageJ (http://www.rsb.info.nih.gov/ij/).

Immunocytochemical analysis. Immunofluorescent staining of cultured $\mathrm{ONH}$ astrocytes was performed as previously described. ${ }^{11}$ Briefly, the cells were fixed with $4 \%$ paraformaldehyde (Sigma)/PBS for $1 \mathrm{~h}$ at room temperature. To prevent nonspecific background, the cells were incubated in $1 \%$ BSA/PBS for $1 \mathrm{~h}$ at room temperature before incubation with mouse monoclonal anti-GFAP antibody (1:300; Sigma) and rabbit polyclonal anti-SOD2 antibody (1:2000; Santa Cruz Biotechnology) for $16 \mathrm{~h}$ at $4^{\circ} \mathrm{C}$. After several wash steps, the cells were incubated with the secondary antibody, Alexa Fluor 488 dye-conjugated goat anti-mouse IgG antibody (1:100; Invitrogen) or Alexa Fluor 488 dye-conjugated goat anti-rabbit IgG antibody (1:100; Invitrogen) for $4 \mathrm{~h}$ at $4{ }^{\circ} \mathrm{C}$ and subsequently washed with PBS. The cells were counterstained with the nucleic acid stain Hoechst $33342(1 \mu \mathrm{g} / \mathrm{ml}$, Life Technologies, Grand Island, NY, USA) in PBS. Images were acquired with confocal microscopy (Olympus FluoView1000; Olympus, Tokyo, Japan).

Morphology analysis for mitochondria. Mitochondria in the $\mathrm{ONH}$ astrocytes were labeled by the addition of a red fluorescent mitochondrial dye to the cultures (100 nM final concentration; MitoTracker Red CMXRos; InvitrogenMolecular Probes) and maintaining it for $20 \mathrm{~min}$ in a $\mathrm{CO}_{2}$ incubator. This dye is concentrated in active mitochondria by a process that is dependent on mitochondrial membrane potential (i.e., accumulation is inhibited by actinomycin A but not by rotenone). The cultures were subsequently fixed with $4 \%$ paraformaldehyde (Sigma) in DPBS for $30 \mathrm{~min}$ at $4{ }^{\circ} \mathrm{C}$ and counterstained with Hoechst $33342(1 \mu \mathrm{g} / \mathrm{ml}$; Invitrogen-Molecular Probes) in DPBS. For a 3D reconstruction, images were obtained with optical section separation (z-interval) of $0.25 \mu \mathrm{m}$ by Olympus FluoView1000. Isosurface rendition was obtained from the stack using Imaris 6.4.2 (Bitplane AG, Zurich, Switzerland).

Electron microscopy. For conventional electron microscopy, cultured ONH astrocytes after treatment were fixed with $2 \%$ paraformaldehyde, $2.5 \%$ glutaraldehyde (Ted Pella, Redding, CA, USA) in $0.15 \mathrm{M}$ sodium cacodylate $(\mathrm{pH}$ 7.4) at $37^{\circ} \mathrm{C}$ and placed in pre-cooled fixative on ice for $1 \mathrm{~h}$. The following procedure was used to optimize mitochondrial structural preservation and membrane contrast. The cells were post-fixed with $1 \%$ osmium tetroxide, $0.8 \%$ potassium ferrocyanide, $3 \mathrm{mM}$ calcium chloride in $0.1 \mathrm{M}$ sodium cacodylate $(\mathrm{pH}$ 7.4) for $1 \mathrm{~h}$, washed with ice-cold distilled water, post-stained with $2 \%$ uranyl acetate at $4{ }^{\circ} \mathrm{C}$, dehydrated using graded ethanols, and embedded in Durcupan resin (Fluka, St. Louis, MO, USA). Ultrathin $(70 \mathrm{~nm})$ sections were post-stained with uranyl acetate and lead salts and evaluated by a JEOL 1200FX (JEOL, Tokyo, Japan) TEM operated at $80 \mathrm{kV}$. Images were recorded on film at $\times 8000$ magnification. The negatives were digitized at $1800 \mathrm{dpi}$ using a Nikon Cool scan system (Nikon Instruments Inc., Melville, NY, USA), giving an image size of $4033 \times 6010$ pixel array and a pixel resolution of $1.77 \mathrm{~nm}$. Mitochondrial lengths were measured with ImageJ. The mitochondrial volume density, defined as the volume occupied by mitochondria divided by the volume occupied by the cytoplasm, was estimated using stereology as follows. A $112 \times 112$ square grid $(112 \times 112$ chosen for ease of use with Photoshop) was overlaid on each image loaded in Photoshop (Adobe Systems Inc., San Jose, CA, USA), and the mitochondria and cytoplasm lying under intercepts were counted. The relative volume of the mitochondria was expressed as the ratio of intercepts coinciding with this organelle relative to the intercepts coinciding with the cytoplasm.

Electron microscope tomography. Sections of cells from each group were cut at thicknesses of $400-500 \mathrm{~nm}$. Sections were then stained $30 \mathrm{~min}$ in $2 \%$ aqueous uranyl acetate, followed by $15 \mathrm{~min}$ in lead salts. Fiducial cues consisting of $20 \mathrm{~nm}$ and colloidal gold particles were deposited on opposite sides of the section. 
For each reconstruction, a series of images at regular tilt increments was collected with a JEOL 4000EX intermediate-voltage electron microscope operated at $400 \mathrm{kV}$. The specimens were irradiated before initiating a tilt series in order to limit anisotropic specimen thinning during image collection. Tilt series were recorded using a $4 \times 4 \mathrm{k}^{2} \mathrm{CCD}$ camera with an angular increment of $2^{\circ}$ from $-60^{\circ}$ to $+60^{\circ}$ about an axis perpendicular to the optical axis of the microscope using a computer-controlled goniometer to increment accurately the angular steps. The illumination was held to near parallel beam conditions and optical density was maintained constant by varying the exposure time. The magnification was 12000 and the pixel resolution was $1.2 \mathrm{~nm}$. The IMOD package (http://bio3d.colorado.edu/imod/) was used for rough alignment with the fine alignment and reconstruction was performed using the TxBR package (National Center for Microscopy and Imaging Research, San Diego, CA, USA). Volume segmentation was performed by manual tracing in the planes of highest resolution with the program Xvoxtrace. ${ }^{58}$ The mitochondrial reconstructions were visualized using Analyze (Mayo Foundation, Rochester, MN, USA) or the surface-rendering graphics of Synu (National Center for Microscopy and Imaging Research, San Diego, CA, USA) as described. ${ }^{58}$ These programs allow one to step through slices of the reconstruction in any orientation and to track or model features of interest in three dimensions.

Cell viability measurement. Cell viability was measured using 3-[4,5-dimethylthiazol-2yl]-2,5-diphenyl tetrazolium bromide (MTT) according to the manufacturer's recommendations (Cell Proliferation Kit 1; Roche Diagnostics, Indianapolis, IN, USA). Briefly, ONH astrocytes were plated on a 96-well plate $\left(1 \times 10^{3}\right.$ per well) and after $24 \mathrm{~h}$, the cells were pre-incubated with $50 \mu \mathrm{g} / \mathrm{ml}$ $\mathrm{CoQ}_{10}$ for $24 \mathrm{~h}$, and then exposed to $\mathrm{H}_{2} \mathrm{O}_{2}$. At various times, a mixture of $100-\mathrm{ml}$ fresh medium and 10- $\mu$ l MTT stock solution was added to each well including the negative control. The cells were incubated for $4 \mathrm{~h}$ in a humidified atmosphere of a $5 \% \mathrm{CO}_{2}$ incubator at $37^{\circ} \mathrm{C}, 100 \mu \mathrm{l}$ of solubilization solution was added per well to resolve the crystallized formazan. After incubation for $16-18 \mathrm{~h}$ in a humidified atmosphere of a $5 \% \mathrm{CO}_{2}$ incubator at $37^{\circ} \mathrm{C}$, the absorbance at $560 \mathrm{~nm}$ was measured with a microplate reader (Spectra MAX; Molecular Devices Corp., Sunnyvale, CA, USA). Each set of data was collected from multiple replicate wells of each experimental group $(n=3)$.

ROS measurement. The intracellular ROS was measured by 5-(and-6)-chloromethyl-2', $7^{\prime}$-dichlorodihydrofluorescein diacetate, acetyl ester (CM- $\mathrm{H}_{2}$ DCFDA, Life Technologies), a chloromethyl derivative of $\mathrm{H}_{2}$ DCFDA, useful as an indicator for ROS in cells. Briefly, ONH astrocytes were plated on a six-well plate $\left(1.7 \times 10^{4}\right.$ per well) and after $24 \mathrm{~h}$, cells were pre-incubated with $50 \mu \mathrm{g} / \mathrm{ml}$ $\mathrm{CoQ}_{10}$ for $24 \mathrm{~h}$, and then exposed to $\mathrm{H}_{2} \mathrm{O}_{2}$. The cells were detached with typsin/ EDTA and loaded with $20 \mu \mathrm{M} \mathrm{CM}-\mathrm{H}_{2}$ DCFDA at $37^{\circ} \mathrm{C}$ for $20 \mathrm{~min}$, and then fluorescence of the sample was measured immediately using flow cytometry (BD FACSCanto II, BD Bioscience, San Diego, CA, USA). Each set of data was collected from multiple replicate dishes of each experimental group $(n=3)$.

Cellular ATP measurement. ATP levels were measured using a luciferasebased assay kit (CellTiter-Glo, Promega Corp., Madison, WI, USA). Briefly, ONH astrocytes were plated on a 96-well plate $\left(1 \times 10^{3}\right.$ per well) and after $24 \mathrm{~h}$, the cells were pre-incubated with $50 \mu \mathrm{g} / \mathrm{ml} \mathrm{CoQ}_{10}$ for $24 \mathrm{~h}$, and then exposed to $\mathrm{H}_{2} \mathrm{O}_{2}$. ATP standard curves from 0 to $1000 \mathrm{nM}$ of ATP in growth media were prepared. After $\mathrm{H}_{2} \mathrm{O}_{2}$ treatment, $100 \mu \mathrm{l}$ growth media was added, followed by $100 \mu \mathrm{l}$ of the CellTiter-Glo reagent per well to lyse cells using vigorous shaking for $2 \mathrm{~min}$ at room temperature. At this point, the prepared ATP standard solution was aded. To stabilize the luminescence signal, cells at room temperature were incubated for $10 \mathrm{~min}$. The luminescence was measured using a microplate luminometer (Labsystems Luminoskan, GMI Inc., Minneapolis, MN, USA). Each set of data was collected from multiple replicate wells of each experimental group $(n=3)$.

Statistical analysis. Data were presented as the mean \pm S.D. or S.E.M. Comparison of two or three experimental conditions was evaluated using the unpaired, two-tailed Student's t-test or one-way analysis of variance and the Bonferroni $t$-test. $P<0.05$ was considered statistically significant.

\section{Conflict of Interest}

The authors declare no conflict of interest.
Acknowledgements. We thank Professor James D Lindsey for critical comments on the manuscript. This work was supported, in part, by NIH Grants EY018658 (WKJ) and NCRR P41 RR004050 and P41GM103412-24 (MHE), and an unrestricted grant from Research to Prevent Blindness (New York, NY, USA).

1. Cadenas E, Davies KJ. Mitochondrial free radical generation, oxidative stress, and aging. Free Radic Biol Med 2000; 29: 222-230

2. Chrysostomou V, Rezania F, Trounce IA, Crowston JG. Oxidative stress and mitochondrial dysfunction in glaucoma. Curr Opin Pharmacol 2013; 13: 12-15

3. McElnea EM, Quill B, Docherty NG, Irnaten M, Siah WF, Clark AF et al. Oxidative stress, mitochondrial dysfunction and calcium overload in human lamina cribrosa cells from glaucoma donors. Mol Vis 2011; 17: 1182-1191.

4. Abu-Amero KK, Morales J, Bosley TM. Mitochondrial abnormalities in patients with primary open-angle glaucoma. Invest Ophthalmol Vis Sci 2006; 47: 2533-2541.

5. Lee D, Kim KY, Noh YH, Chai S, Lindsey JD, Ellisman MH et al. Brimonidine blocks glutamate excitotoxicity-induced oxidative stress and preserves mitochondrial transcription factor a in ischemic retinal injury. PLOS One 2012; 7: e47098.

6. Hernandez MR, Agapova OA, Yang P, Salvador-Silva M, Ricard CS, Aoi S. Differential gene expression in astrocytes from human normal and glaucomatous optic nerve head analyzed by cDNA microarray. Glia 2002; 38: 45-64.

7. Weinreb RN, Khaw PT. Primary open-angle glaucoma. Lancet 2004; 363: 1711-1720.

8. Hernandez MR. The optic nerve head in glaucoma: role of astrocytes in tissue remodeling. Prog Retin Eye Res 2000; 19: 297-321.

9. Tezel G. Oxidative stress in glaucomatous neurodegeneration: mechanisms and consequences. Prog Retin Eye Res 2006; 25: 490-513.

10. Dai C, Khaw PT, Yin ZQ, Li D, Raisman G, Li Y. Structural basis of glaucoma: the fortified astrocytes of the optic nerve head are the target of raised intraocular pressure. Glia 2012; 60: 13-28.

11. Ju WK, Kim KY, Lindsey JD, Angert M, Duong-Polk KX, Scott RT et al. Intraocular pressure elevation induces mitochondrial fission and triggers OPA1 release in glaucomatous optic nerve. Invest Ophthalmol Vis Sci 2008; 49: 4903-4911.

12. Ju WK, Kim KY, Angert M, Duong-Polk KX, Lindsey JD, Ellisman MH et al. Memantine blocks mitochondrial OPA1 and cytochrome $c$ release and subsequent apoptotic cell death in glaucomatous retina. Invest Ophthalmol Vis Sci 2009; 50: 707-716.

13. Ju WK, Kim KY, Duong-Polk KX, Lindsey JD, Ellisman MH, Weinreb RN. Increased optic atrophy type 1 expression protects retinal ganglion cells in a mouse model of glaucoma. Mol Vis 2010; 16: 1331-1342.

14. Son JL, Soto I, Oglesby E, Lopez-Roca T, Pease ME, Quigley HA et al. Glaucomatous optic nerve injury involves early astrocyte reactivity and late oligodendrocyte loss. Glia 2010; 58: 780-789.

15. Beal MF, Shults CW. Effects of Coenzyme Q10 in Huntington's disease and early Parkinson's disease. Biofactors 2003; 18: 153-161.

16. McCarthy S, Somayajulu M, Sikorska M, Borowy-Borowski H, Pandey S. Paraquat induces oxidative stress and neuronal cell death; neuroprotection by water-soluble coenzyme Q10. Toxicol Appl Pharmacol 2004; 201: 21-31.

17. Bessero AC, Clarke PG. Neuroprotection for optic nerve disorders. Curr Opin Neurol 2010; 23: $10-15$.

18. Qu J, Kaufman $\mathrm{Y}$, Washington I. Coenzyme Q10 in the human retina. Invest Ophthalmol Vis Sci 2009; 50: 1814-1818

19. Nucci C, Tartaglione R, Cerulli A, Mancino R, Spano A, Cavaliere F et al. Retinal damage caused by high intraocular pressure-induced transient ischemia is prevented by coenzyme Q10 in rat. Int Rev Neurobiol 2007; 82: 397-406.

20. Russo R, Cavaliere F, Rombola L, Gliozzi M, Cerulli A, Nucci C et al. Rational basis for the development of coenzyme Q10 as a neurotherapeutic agent for retinal protection. Prog Brain Res 2008; 173: 575-582.

21. Nakajima $Y$, Inokuchi $Y$, Nishi M, Shimazawa M, Otsubo K, Hara H. Coenzyme Q10 protects retinal cells against oxidative stress in vitro and in vivo. Brain Res 2008; 1226: 226-233.

22. Lulli M, Witort E, Papucci L, Torre E, Schipani C, Bergamini C et al. Coenzyme Q10 instilled as eye drops on the cornea reaches the retina and protects retinal layers from apoptosis in a mouse model of kainate-induced retinal damage. Invest Ophthalmol Vis Sci 2012; 53: 8295-8302.

23. Hernandez MR, Igoe F, Neufeld AH. Cell culture of the human lamina cribrosa. Invest Ophthalmol Vis Sci 1988; 29: 78-89.

24. Yu AL, Fuchshofer R, Birke M, Kampik A, Bloemendal H, Welge-Lussen U. Oxidative stress and TGF-beta2 increase heat shock protein 27 expression in human optic nerve head astrocytes. Invest Ophthalmol Vis Sci 2008; 49: 5403-5411.

25. Hernandez MR, Miao H, Lukas T. Astrocytes in glaucomatous optic neuropathy. Prog Brain Res 2008; 173: 353-373.

26. John GB, Shang Y, Li L, Renken C, Mannella CA, Selker JM et al. The mitochondrial inner membrane protein mitofilin controls cristae morphology. Mol Biol Cell 2005; 16 : 1543-1554.

27. Van Laar VS, Dukes AA, Cascio M, Hastings TG. Proteomic analysis of rat brain mitochondria following exposure to dopamine quinone: implications for Parkinson disease. Neurobiol Dis 2008; 29: 477-489. 
28. Marriage BJ, Clandinin MT, Macdonald IM, Glerum DM. Cofactor treatment improves ATP synthetic capacity in patients with oxidative phosphorylation disorders. Mol Genet Metab 2004; 81: 263-272.

29. Haas $\mathrm{RH}$. The evidence basis for coenzyme $Q$ therapy in oxidative phosphorylation disease. Mitochondrion 2007; 7(Suppl): S136-S145.

30. Fragaki K, Cano A, Benoist JF, Rigal O, Chaussenot A, Rouzier $\mathrm{C}$ et al. Fatal heart failure associated with COQ10 and multiple OXPHOS deficiency in a child with propionic acidemia. Mitochondrion 2011; 11: 533-536.

31. He Y, Leung KW, Zhang YH, Duan S, Zhong XF, Jiang RZ et al. Mitochondrial complex I defect induces ROS release and degeneration in trabecular meshwork cells of POAG patients: protection by antioxidants. Invest Ophthalmol Vis Sci 2008; 49: 1447-1458.

32. Malone PE, Hernandez MR. 4-Hydroxynonenal, a product of oxidative stress, leads to an antioxidant response in optic nerve head astrocytes. Exp Eye Res 2007; 84: 444-454.

33. Mozaffarieh M, Flammer J. Is there more to glaucoma treatment than lowering IOP? Surv Ophthalmol 2007; 52(Suppl 2): S174-S179.

34. Mozaffarieh M, Flammer J. A novel perspective on natural therapeutic approaches in glaucoma therapy. Expert Opin Emerg Drugs 2007; 12: 195-198.

35. Mozaffarieh M, Grieshaber MC, Orgul S, Flammer J. The potential value of natural antioxidative treatment in glaucoma. Surv Ophthalmol 2008; 53: 479-505.

36. Lee S, Sheck L, Crowston JG, Van Bergen NJ, O'Neill EC, O'Hare F et al. Impaired complex-I-linked respiration and ATP synthesis in primary open-angle glaucoma patient lymphoblasts. Invest Ophthalmol Vis Sci 2012; 53: 2431-2437.

37. Yu-Wai-Man P, Griffiths PG, Chinnery PF. Mitochondrial optic neuropathies-disease mechanisms and therapeutic strategies. Prog Retin Eye Res 2011; 30: 81-114.

38. Yu AL, Moriniere J, Birke M, Neumann C, Fuchshofer R, Kampik A et al. Reactivation of optic nerve head astrocytes by TGF-beta2 and $\mathrm{H} 2 \mathrm{O} 2$ is accompanied by increased $\mathrm{Hsp} 32$ and Hsp47 expression. Invest Ophthalmol Vis Sci 2009; 50: 1707-1717.

39. Shults CW, Haas RH, Passov D, Beal MF. Coenzyme Q10 levels correlate with the activities of complexes I and II/III in mitochondria from parkinsonian and nonparkinsonian subjects. Ann Neurol 1997; 42: 261-264.

40. Ferrante RJ, Andreassen OA, Dedeoglu A, Ferrante KL, Jenkins BG, Hersch SM et al. Therapeutic effects of coenzyme Q10 and remacemide in transgenic mouse models of Huntington's disease. J Neurosci 2002; 22: 1592-1599.

41. Guo L, Cordeiro MF. Assessment of neuroprotection in the retina with DARC. Prog Brain Res 2008; 173: 437-450.

42. Lulli M, Witort E, Papucci L, Torre E, Schiavone N, Dal Monte M et al. Coenzyme Q10 protects retinal cells from apoptosis induced by radiation in vitro and in vivo. J Radiat Res 2012; 53: 695-703

43. Rohl C, Armbrust E, Kolbe K, Lucius R, Maser E, Venz S et al. Activated microglia modulate astroglial enzymes involved in oxidative and inflammatory stress and increase the resistance of astrocytes to oxidative stress in vitro. Glia 2008; 56: 1114-1126.

44. Bruce-Keller AJ, Geddes JW, Knapp PE, McFall RW, Keller JN, Holtsberg FW et al. Anti-death properties of TNF against metabolic poisoning: mitochondrial stabilization by MnSOD. J Neuroimmunol 1999; 93: 53-71.
45. Wu Z, Puigserver P, Andersson U, Zhang C, Adelmant G, Mootha V et al. Mechanisms controlling mitochondrial biogenesis and respiration through the thermogenic coactivator PGC-1. Cell 1999; 98: 115-124.

46. Lin J, Wu H, Tarr PT, Zhang CY, Wu Z, Boss $O$ et al. Transcriptional co-activator PGC-1 alpha drives the formation of slow-twitch muscle fibres. Nature 2002; 418: 797-801.

47. Larsson NG, Wang J, Wilhelmsson $\mathrm{H}$, Oldfors $\mathrm{A}$, Rustin $\mathrm{P}$, Lewandoski $\mathrm{M}$ et al. Mitochondrial transcription factor $A$ is necessary for mtDNA maintenance and embryogenesis in mice. Nat Genet 1998; 18: 231-236.

48. Scarpulla RC. Transcriptional activators and coactivators in the nuclear control of mitochondrial function in mammalian cells. Gene 2002; 286: 81-89.

49. Fosslien E. Mitochondrial medicine-molecular pathology of defective oxidative phosphorylation. Ann Clin Lab Sci 2001; 31: 25-67.

50. Bargossi AM, Battino M, Gaddi A, Fiorella PL, Grossi G, Barozzi G et al. Exogenous CoQ10 preserves plasma ubiquinone levels in patients treated with 3-hydroxy-3-methylglutaryl coenzyme A reductase inhibitors. Int J Clin Lab Res 1994; 24: 171-176.

51. Fukui $\mathrm{H}$, Moraes $\mathrm{CT}$. The mitochondrial impairment, oxidative stress and neurodegeneration connection: reality or just an attractive hypothesis? Trends Neurosci 2008; 31: 251-256.

52. Balaban RS, Nemoto S, Finkel T. Mitochondria, oxidants, and aging. Cell 2005; 120 : 483-495.

53. Carelli V, Rugolo M, Sgarbi G, Ghelli A, Zanna C, Baracca A et al. Bioenergetics shapes cellular death pathways in Leber's hereditary optic neuropathy: a model of mitochondrial neurodegeneration. Biochim Biophys Acta 2004; 1658: 172-179.

54. Beal MF. Aging, energy, and oxidative stress in neurodegenerative diseases. Ann Neurol 1995; 38: 357-366.

55. Bergamini C, Moruzzi N, Sblendido A, Lenaz G, Fato R. A water soluble CoQ10 formulation improves intracellular distribution and promotes mitochondrial respiration in cultured cells. PLoS One 2012; 7: e33712.

56. Quinzii CM, Lopez LC, Gilkerson RW, Dorado B, Coku J, Naini AB et al. Reactive oxygen species, oxidative stress, and cell death correlate with level of CoQ10 deficiency. FASEB J 2010; 24: 3733-3743.

57. Fernandez-Marcos PJ, Auwerx J. Regulation of PGC-1alpha, a nodal regulator of mitochondrial biogenesis. Am J Clin Nutr 2011; 93: 884S-890S.

58. Perkins GA, Renken CW, Song JY, Frey TG, Young SJ, Lamont S et al. Electron tomography of large, multicomponent biological structures. J Struct Biol 1997; 120: 219-227.

(i) $(-)$ Cell Death and Disease is an open-access journal published by Nature Publishing Group. This work is licensed under a Creative Commons Attribution-NonCommercialNoDerivs 3.0 Unported License. To view a copy of this license, visit http://creativecommons.org/licenses/by-nc-nd/3.0/ 